Les sources de l'Histoire des Mines : Nouveaux outils, Nouvelles approches

\title{
Les sources de l'histoire minière aux Archives départementales de la Loire
}

The archives of the history of mining in the Archives départementales de la Loire

Denys Barau

\section{(2) OpenEdition}

\section{Journals}

Édition électronique

URL : http://journals.openedition.org/dht/633

DOI : $10.4000 /$ dht.633

ISSN : $1775-4194$

Éditeur :

Centre d'histoire des techniques et de l'environnement du Cnam (CDHTE-Cnam), Société des élèves du CDHTE-Cnam

Édition imprimée

Date de publication : 1 décembre 2008

Pagination : 40-66

ISBN : 978-2-95-30779-2-6

ISSN : 0417-8726

Référence électronique

Denys Barau, «Les sources de l'histoire minière aux Archives départementales de la Loire », Documents pour l'histoire des techniques [En ligne], 16 | $2^{\mathrm{e}}$ semestre 2008, mis en ligne le 08 novembre 2010, consulté le 08 septembre 2020. URL : http://journals.openedition.org/dht/633 ; DOI : https:// doi.org/10.4000/dht.633 


\title{
Les sources de l'histoire minière aux Archives départementales de la Loire
}

\author{
Denys Barau \\ Archives départementales de la Loire
}

\begin{abstract}
RÉSUMÉ
Élément important de la révolution industrielle à l'échelle nationale dans la première moitié du XIXe siècle, l'extraction du charbon a durablement marqué la région stéphanoise, non seulement dans son développement économique, mais aussi dans son peuplement, sa vie sociale, son paysage et sa culture. Le régime de la concession institué par la loi de 1810 explique que l'histoire de cette industrie trouve son matériau principal dans deux fonds d'archives distincts : celui des entreprises minières (compagnies privées d'abord, puis, à partir de 1945, Houillères du bassin de la Loire nationalisées) et celvi de l'administration des Mines, chargée de contrôler l'exploitation. Mais à ce bloc central vient s'ajouter une grande diversité de sources complémentaires, mêlant archives publiques et archives privées, imprimés ou iconographie, qui éclairent certains moments forts de l'histoire minière stéphanoise et ses dimensions techniques, économiques et humaines.
\end{abstract}

Résumés et mots clés en anglais sont regroupés en fin de volume, accompagnés des mots clés français

$P^{\prime}$ incipal producteur français de charbon dans la première moitié du XIXe siècle, le bassin stéphanois a constitué un élément important pour le démarrage de la révolution industrielle à l'échelle du pays. Sur le plan local plus particulièrement, cette production houillère a été l'un des principaux facteurs d'un développement remarquable de l'industrie. Les fonds relatifs à l'histoire minière occupent aux Archives départementales de la Loire une place à la mesure de ce rôle essentiel. Afin d'éclairer la description que nous nous proposons d'en faire, nous fixerons d'abord quelques repères sur l'histoire de l'exploitation elle-même. Nous présenterons ensuite les pièces centrales de cet ensemble : les archives spécifiques que forment complémentairement les fonds des entreprises minières d'un côté, et celles de l'administration des Mines de l'autre. En dehors de ces séries, il existe sur la question d'autres ressources documentaires, dont certaines sont loin d'être négligeables; nous en donnerons pour finir un aperçu.
Brève présentation de l'exploitation minière dans la Loire Il s'agit principalement ici du bassin hoviller stéphanois, mais il convient de rappeler que s'il a été, et de très loin, la plus importante exploitation minière du département, il n'a pas été la seule. Des gisements métallifères d'abord ont été exploités à différentes époques : du cuivre au XVe siècle, dans les Monts du Lyonnais, où Jacques Cœur possédait des mines; de I'or, dit-on, au début du XVIIe siècle, à Saint-Martin-laPlaine ; du plomb entre le début du XVIIIe siècle et les années 1840, dans le massif du Pilat (Saint-Julien-MolinMolette) et les Monts du Forez (autour de Saint-Martinla-Sauveté), pour le compte d'une famille d'origine autrichienne, les Kayr de Blumenstein. Récemment, entre 1955 et les années 1980, on a extrait de l'uranium à Saint-Priest-la-Prugne. Des filons d'anthracite ont également été exploités jusqu'un peu avant la seconde Guerre mondiale, dans le Roannais (autour

1 L'exposé qui suit est largement tributaire des études déjà réalisées sur l'histoire des mines de la Loire, dont on trouvera un inventaire sommaire en conclusion du présent article. 
de Bully et de Saint-Symphorien-de-Lay), avec une production annuelle de l'ordre de quelques milliers de tonnes (23 500 tonnes à Bully, la meilleure année de production, en 1908).

Rien de tout cela, répétons-le, n'a approché pourtant l'ampleur de la production hovillère de la région stéphanoise, ni l'importance de ses effets. Le bassin s'étend sur une superficie approximative de 22000 ha, entre Rive-de-Gier à l'Est et Firminy à l'Ouest, et s'articule en trois secteurs distincts : celui du Gier, celui de Saint-Étienne et celui de l'Ondaine. Attestée dès le XIVe siècle, l'exploitation n'a commencé à s'intensifier que dans les années 1720 et surtout à partir du début du XIXe siècle, en commençant par le secteur le plus oriental, celui de Rive-de-Gier, pour s'étendre progressivement vers l'Ouest. Entre les années 1820 et 1873, la production a connu une croissance rapide, passant de 350000 tonnes à 4 millions; elle a ensuite oscillé autour de ce dernier chiffre jusqu'en 1939, avec un maximum (4,9 millions) en 1918. D'abord très morcelée - dans les années 1820, on comptait au moins une quarantaine d'entreprises pour le seul secteur de Rive-de-Gier - l'exploitation a connu une première concentration en octobre 1837 avec la formation de la Compagnie générale des mines de Rive-de-Gier regroupant deux petites entreprises d'extraction, la compagnie du Logis-des-Pères et celle du GourdMarin ; des fusions successives ont abouti au début de 1844 à la création de la Compagnie générale des mines de la Loire, qui, après avoir étendu son activité à la partie stéphanoise du bassin, devint à l'automne de l'année suivante la Compagnie des mines de la Loire. Sous la conduite d'un capitaine d'industrie d'inspiration saint-simonienne, Gustave Delahante 2, le bassin entrait alors dans l'ère de la grande entreprise capitaliste. Elle ne réunissait qu'un peu plus de la moitié des concessions (33 sur 60) et un peu moins du tiers du territoire minier (5 979 ha), mais c'était la partie la plus activement exploitée, dont on avait extrait en 1844 plus d'un million de tonnes, contre 185000 pour

2 Né en 1816, mort en 1905, il était issu d'une famille de fermiers généraux ; son père, Adrien Delahante, banquier important sur la place de Lyon, était administrateur de la Compagnie du canal de Givors. Après la dissolution de la Compagnie des mines de la Loire, il devait être associé au duc de Morny dans la compagnie de chemin de fer du Grand Central, puis participer à la fondation de la Banque de Paris et des Pays-Bas (voir Pierre Guillaume, La Compagnie des Mines de la Loire. 1846-1854. Essai sur l'apparition de la grande industrie capitaliste en France, Paris, Presses universitaires de France, 1966, pp.63-64). le reste du bassin ${ }^{3}$. Les compagnies «dissidentes », dont celle de Roche-la-Molière et Firminy, créée en 1820, qui contrôlait dans la partie occidentale la concession la plus vaste, ne représentaient donc qu'une concurrence très limitée et les nombreux adversaires de cette nouvelle compagnie ont pu avec quelque apparence dénoncer en elle un « monopole ». Son existence fut en effet la cible d'une polémique vigoureuse, non seulement sur le plan local, mais aussi au niveau national : dans un discours à la Chambre des députés, le 24 mars 1846, Lamartine devait s'en prendre à la monopolisation des « entrailles même de la terre... [des] sources du feu, [des] réservoirs que la Providence avait disposés [...] pour servir à la consommation de la nation pendant des siècles 1 . Finalement, le 17 octobre 1854, Napoléon III mit un terme à l'expérience en imposant le fractionnement de la compagnie en quatre sociétés : la Société anonyme des Mines de la Loire, les Hovillères de Saint-Étienne, la Compagnie des mines de Montrambert et de La Béraudière et les Houillères de Rive-de-Gier. L'exploitation dans la partie orientale du bassin ayant rapidement décliné, cette dernière société fut mise en liquidation en 1911. II n'y avait désormais plus de « monopole », mais la production restait dominée par quelques grandes sociétés, sans toutefois que la petite exploitation ait été entièrement éliminée. On comptait encore 17 entreprises en 1913 ; sur les 11 qui restaient en 1935, 5 produisaient entre 200000 et un million de tonnes par an, 3 entre 20000 et 60000 tonnes et la production d'une seule des 3 autres atteignait les 1000 tonnes ${ }^{4}$.

L'ensemble ayant été nationalisé par la loi du 17 mai 1946, un décret de juin de la même année créa les Hovillères du Bassin de la Loire (HBL). Réorganisée, l'exploitation cessa entièrement dans la partie orientale du bassin à partir de 1950 et se concentra dans la partie occidentale ; la production ne marqua d'abord jusqu'en 1960 qu'un léger fléchissement, en passant de 4 millions de tonnes annuelles à 3. Le déclin devait s'accélérer ensuite, avec une production de 1, 5 mil-

3 P. Guillaume, op.cit. note 2, p.42.

4 Pour la première catégorie : Roche-la-Molière et Firminy (954 493), Mines de la Loire (826 290), Montrambert et La Béraudière (707 160), Houillères de Saint-Étienne (444 728) et Houillère de La Chazotte (240 328). Pour la deuxième : Mines de La Péronnière (68 056), Mines du Cros (62 497), Houillères de Saint-Chamond (40 375). Pour le troisième: Mines de La Montagne-du-feu (1 040), Mines de Combeplaine (990) et Mines de La Catonière (165). Ces informations sont données par Maxime Perrin, Saint-Étienne et sa région économique. Un type de la vie industrielle en France, Tours, Arrault et Cie, 1937, p.102. 


\section{Les sources de l'histoire minière aux Archives départementales de la Loire}

lions de tonnes en 1970, de moins de 500000 en 1975 et de 150000 en 1980. Parallèlement la main d'œuvre se réduisait aussi : on comptait encore 22000 mineurs en 1946 ; ils n'étaient plus que 730 en 1979. Initialement prévue pour 1975, la fin de l'exploitation fut reportée à cause du choc pétrolier, repoussée de nouveau en 1980, et c'est seulement le 1er juillet 1983 que le dernier puits, le puits Pigeot à La Ricamarie, a cessé de fonctionner.

Ces quelques deux siècles d'exploitation hovillère n'ont pas manqué d'avoir des conséquences importantes et multiformes sur la région stéphanoise. Bien que certaines de ses industries les plus caractéristiques, la rubanerie ou l'armurerie par exemple, en aient été assez peu tributaires, un pan essentiel de son développement est né de la présence locale du charbon : la métallurgie lourde et la sidérurgie d'abord avec quelques entreprises phares comme les Forges et aciéries de la Marine et des Chemins de fer à Saint-Chamond, ou les Établissements Jacob-Holtzer à Unieux ; plusieurs d'entre elles ont formé dans les années 1950 la Compagnie des ateliers et forges de la Loire (CAFL), puis, en fusionnant avec la Société des forges et aciéries du Creusot en 1970, le groupe Creusot-Loire. Le charbon a également été le facteur déterminant pour l'implantation dans la région d'une autre industrie, la verrerie, qui a eu une présence très massive à Rive-de-Gier avant d'essaimer dans d'autres localités du département où elle a jusqu' à aujourd'hui mieux résisté (SaintJust-sur-Loire, Veauche, Saint-Romain-le-Puy). La mine a pesé aussi sur le peuplement de la région : en 1826 , 2700 personnes y étaient employées; elles étaient 6300 vingt ans après, et plus de 18000 en 1898. Elle a attiré toute une partie de l'excédent humain des campagnes environnantes, et à partir de la guerre de 1914-1918, une main d'œuvre coloniale ou étrangère, qui représentait en 1935 plus du quart des quelques 16000 salariés du bassin 5 . La marque de la mine sur la région semble toutefois avoir été moins forte, moins exclusive surtout, que dans d'autres régions. S'il y a eu, par exemple, des cités minières, elles se sont généralement assez bien fondues dans le tissu urbain et n'ont jamais présenté l'ampleur, ni la spécificité des corons du Nord. Quelques crassiers dominent encore le paysage, mais des très nombreux chevalements qu' on y a vus longtemps, il ne reste pratiquement plus de trace. Un seul est encore debout : il fait partie d'un musée de

5 Un peu moins de 4800, parmi lesquels plus de 2000 Polonais, un peu moins de 700 Marocains et autant d'Italiens, plus de 400 Espagnols, près de 300 Algériens, une centaine de Portugais et de Grecs. (Voir M. Perrin, op.cit., note 4, pp.104105). la Mine, dont la création a été décidée avant même la fermeture du dernier puits par la municipalité stéphanoise que présidait alors un ancien mineur, Joseph Sanguedolce ; pareille décision témoignait d'une volonté de préserver, à défaut de l'exploitation elle-même, sa mémoire. On pourra voir un autre symbole de cette persistance dans le fait qu'aujourd'hui encore, plus de vingt ans après la fermeture, un feu d'artifice soit encore tiré, tous les 4 décembre, pour célébrer la fête de la Sainte Barbe.

\section{Le bloc documentaire central : archives des entreprises houillères, archives de l'administration des Mines}

Mais si l'on ne veut pas se contenter de préserver un patrimoine, d'entretenir une mémoire, si l'on veut écrire l'histoire de cette exploitation minière, c'est d'abord aux archives qu'il faut avoir recours, et en premier lieu à celles qui ont été spécifiquement produites en relation avec cette exploitation. Conséquence logique de la loi du 21 avril 1810 qui régit en France l'exploitation minière, ces archives sont de deux ordres différents. La loi prévoyait en effet, d'une part, que le droit d'exploiter soit attribué, à titre de «propriété perpétuelle » et dans le cadre d'une " concession » délimitée, à des particuliers ; elle réservait, d'autre part, à l'administration non seulement la définition des concessions et la désignation des titulaires, mais encore, selon les termes de son titre $V$, la surveillance de l'usage qui en était fait. Partant, toute exploitation minière engendre en principe deux ensembles d'archives complémentaires : celui des entreprises exploitantes et celui de l'administration qui les contrôle. Nous décrirons donc ce que représentent ces deux ensembles dans le cas de la Loire.

Les archives des entreprises hovillères

En janvier 1973, alors que l'arrêt de l'exploitation s'annonçait imminent, une partie importante des archives des Houillères du Bassin de la Loire a été transférée aux Archives départementales ; il s'agissait de plus de 3000 articles, jusqu'alors entreposés dans les locaux de l'ancien hôpital du Montcel, à La Ricamarie. Plusieurs autres, d'importance inégale, ont ensuite complété ce versement initial : environ 350 articles en décembre 1974, 3500 autres en mai 1984 ; en 1999, une très volumineuse collection de plans de machines qui avait été emmagasinés au Musée de la Mine a été récupérée par les Archives; des documents ont encore été versés en février 2005 par les Houillère de bassin du Centre et du Midi. L'ensemble, sans compter les 
plans, occupe environ 540 m.l. ${ }^{6}$. II convient par ailleurs de signaler que ce fonds n'est pas absolument complet. Certains types de documents d'abord n'ont pas été versés : une partie des archives du personnel qui ont été confiées à l'Association nationale de gestion des retraites (ANGR), les archives médicales conservées par le médecin régional des mines et les archives techniques (plans du sous-sol, registres d'exploitation par puits) qui ont été prises en charge par le Bureau des recherches géologiques et minières (BRGM). II y a eu également des destructions non contrôlées (lors de la démolition de l'ancien hôpital du Montcel en particulier) ; enfin, certains documents restent probablement encore entre les mains de particuliers.

Lorsque le premier de ces versements a eu lieu, à I'initiative de la directrice des Archives, Éliane Viallard, c'était une expérience tout à fait neuve. D'où quelques incertitudes, sur le statut juridique notamment : on a signé en janvier 1974 un contrat de dépôt comme pour des archives privées. Eł le fonds, logiquement, a aussi trouvé d'abord sa place dans la série J, réservée à ces mêmes archives privées (sous-série $15 \mathrm{~J}$ ), rien n'étant alors prévu dans le cadre de classement des archives départementales pour le cas des entreprises ou des établissements publics. La composition du fonds n'était pas non plus, il est vrai, sans ambiguïté à cet égard : aux archives de l'entreprise nationalisée, les Houillères du Bassin de la Loire, étaient jointes celles des différentes compagnies privées dont elles étaient l'héritière et continuatrice.

Un récolement sommaire des articles composant le premier versement avait été effectué au préalable. Les versements suivants, à l'exception des plans récupérés au Musée de la Mine, ont fait l'objet de bordereaux de versement. En 2000, on a entrepris de classer et d'inventorier l'ensemble du fonds, désormais inscrit dans la série ETP prévue pour les archives des établissements et organismes publics par la circulaire du 18 décembre 1998 (sous-série 1 ETP). II a paru préférable en effet de conserver à l'ensemble son unité plutôt que dissocier les archives de l'entreprise nationalisée (relevant de la série ETP) et celles des compagnies pri-

6 Depuis décembre 2003, un autre fonds minier est conservé aux Archives de la Loire, celui des Houillères du bassin du Centre et du Midi (HBCM) (sous-série 7 ETP). II s'agit en fait des archives des services centraux de cet établissement public, aujourd'hui disparu, qui avait son siège à Saint-Étienne mais gérait des exploitations extérieures au département de la Loire (Saône-et-Loire, Puy-de-Dôme, Isère, Gard, Bouchesdu-Rhône, Tarn...). Comme il ne concerne que très marginalement les mines de la Loire, nous nous contenterons ici de mentionner son existence. vées (en série J). À ce jour, seules les archives des plus importantes de ces compagnies ont été classées de façon définitive : celles de la Compagnie des mines de Roche-la-Molière et Firminy, de la Compagnie des Mines de la Loire, de la Société anonyme des Mines de la Loire, des Houillères de Saint-Étienne et de la Compagnie de Montrambert et de La Béraudière. Soit 6000 articles et environ 250 m.l. Restent à traiter les archives de quelques compagnies moins importantes, celles de quelques sociétés filiales et celles d'organismes professionnels comme le Comité des hovillères, pour cette première période d'exploitation privée, et les archives propres des Houillères du bassin de la Loire, pour la période suivante.

Nous n'évoquerons ici que cette partie classée du fonds, celle dont nous avons, par là même, la connaissance la meilleure. Eł nous n'en décrirons que les caractéristiques principales et les grandes masses. Un examen rapide des inventaires suffit pour constater d'abord un certain déséquilibre sur le plan chronologique : la plus grande partie des documents date de la période comprise entre 1870 et 1945, c'est-à-dire, nous venons de le voir, un moment où le dynamisme de l'exploitation avait déjà un peu faibli ; les années de plus forte croissance, celles où le bassin était le plus important en France, sont beaucoup moins bien représentées. À vrai dire, il est assez rare que ces années là, entre 1840 et 1870 , le soient dans les fonds industriels. Entre les différentes compagnies, le déséquilibre est moins marqué. Toutefois les archives de trois d'entre elles (Roche-la-Molière et Firminy, Mines de la Loire, Montrambert et La Béraudière) sont manifestement plus complètes que celles des deux autres (Compagnie des Mines de la Loire, Houillères de Saint-Étienne). Le fonds le plus incomplet, celui des Houillères de Saint-Étienne, compte seulement 140 articles; il en existe à peu près autant dans celui de la Compagnie des Mines de la Loire, mais pour une dizaine d'années d'existence seulement. On observe aussi une disproportion entre le fonds de Roche-la-Molière et Firminy (un peu plus de 900 articles) et les deux autres (2 070 pour les Mines de la Loire, 2800 pour Montrambert) ; elle s'explique par la seule existence dans ces deux derniers de longues séries de livres de paye et de registres de présence (respectivement 1200 articles et 2 200) ; si l'on met à part ces deux séries, ces trois fonds sont quantitativement presque équivalents, celvi de la compagnie de Montrambert étant un peu moins important 1600 articles environ contre 870-900 pour les autres). II faut signaler aussi l'existence de quelques articles concernant des regroupements d'exploitation antérieurs à 


\section{Les sources de l'histoire minière aux Archives départementales de la Loire}

la Compagnie des Mines de la Loire ( $C^{i e}$ générale des mines de Rive-de-Gier, Société des houillères réunies de Montrambert et de Quartier-Gaillard, Société des mines réunies de Saint-Étienne) et de quelques autres témoignant de l'activité entre 1855 et 1906 d'une " commission des huit délégués des quatre sociétés issues du fractionnement $"$.

La répartition des archives entre les principales fonctions de l'entreprise se présente de la façon suivante (les chiffres représentent le nombre d'articles concernés) :

\begin{tabular}{|c|c|c|c|c|c|}
\hline & 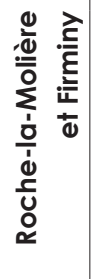 & 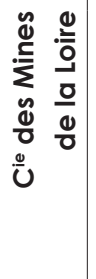 & 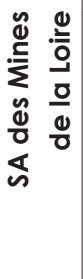 & 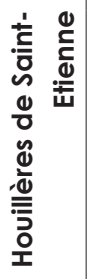 & 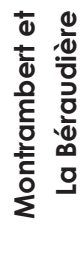 \\
\hline Société & 74 & 33 & 65 & 13 & 24 \\
\hline Direction générale & 216 & 32 & 301 & 20 & 87 \\
\hline Comptabilité & 182 & 49 & 807 & 18 & 1282 \\
\hline Services financiers & 28 & 0 & 76 & 40 & 13 \\
\hline Personnel & 231 & 35 & 652 & 17 & 1217 \\
\hline OEuvres sociales & 30 & 0 & 5 & 3 & 22 \\
\hline Domaine & 40 & 4 & 15 & 3 & 54 \\
\hline Exploitation & 99 & 11 & 140 & 24 & 45 \\
\hline Commercialisation & 6 & 0 & 13 & 2 & \\
\hline
\end{tabular}

L'écrasante disproportion entre les archives fonctionnelles et les archives opérationnelles au bénéfice des premières est ce qui ressort de ce tableau de la façon la plus saillante. Parmi celles de la première catégorie, les archives de société, généralement très complètes, offrent, à travers les séries de procès-verbaux des conseils d'administrations et de rapports aux assemblées générales, une vue d'ensemble sur l'activité de ces compagnies pendant près d'un siècle. Seules, les Houillères de Saint-Étienne font sur ce point exception : nous n'avons de traces des conseils d'administration que pour deux courtes périodes (1927-1929 et 1941-1946), sans que nous puissions expliquer l'origine de cette lacune que compense un peu une série complète de "rapports et décisions» des assemblées générales.

La situation en revanche est beaucoup plus contrastée s'agissant des archives de direction (ill.1) aussi bien du point de vue de leur quantité que de leur composition. Leur importance nettement plus grande pour la Compagnie de Roche-la-Molière et Firminy et pour la Société anonyme des Mines de la Loire s'explique en partie par la présence de longues séries de

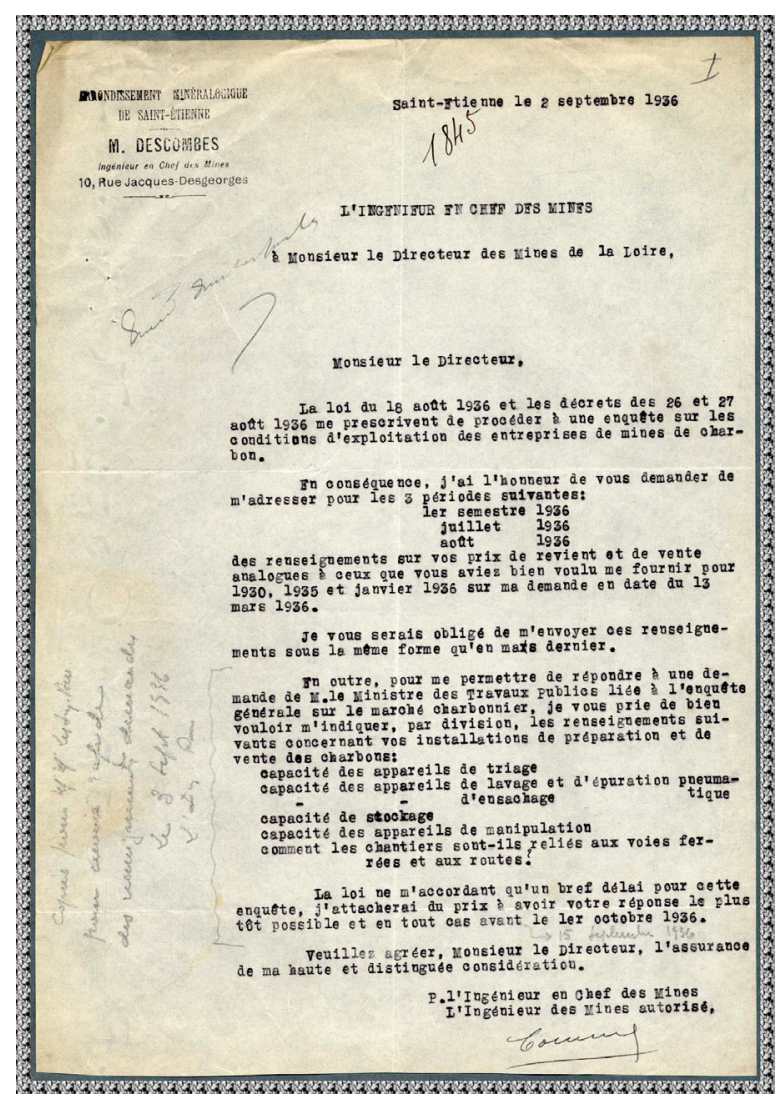

1. Lettre de l'ingénieur en chef des Mines au directeur des Mines de la Loire, Saint-Étienne, 2 septembre $1936(21 \times 29$ $\mathrm{cm}$, AD Loire, 1 ETP 1062

copies de lettres (entre 1853 et 1946 pour RMF et entre 1854 et 1934 pour les Mines de la Loire). C'est aussi une série de ce genre qui constitue le plus clair de ces archives-là pour la $C^{i e}$ des Mines de la Loire. Les dossiers de contentieux ont en revanche été conservés en plus grand nombre pour la SA des Mines de la Loire : ils concernent des problèmes de droit d'exploitation, des accidents du travail, et, chose particulièrement importante pour une exploitation dans une zone très urbanisée, les dégâts de surface. II faut signaler enfin, dans cette catégorie, une collection de rapports annuels des ingénieurs divisionnaires (SA des Mines de la Loire, 1911-1921) et des séries, parfois longues, de notes de service (1874-1946 pour RMF ; 1906-1945 pour SA des Mines de la Loire ; 1930-1946 pour Montrambert).

Les archives comptables sont dans l'ensemble moins abondantes et moins complètes que d'ordinaire dans les fonds industriels. Sur ce plan-là aussi le fonds des Houillères de Saint-Étienne est particulièrement déficitaire; pour la Compagnie des Mines de la Loire, nous n'avons qu'un seul volume du journal général, correspondant à un an et demi d'activité, et la série plus nombreuse de journaux « main d'œuvre 
et fournitures " pour certains puits ou ateliers ne compense pas vraiment cette lacune. Nous avons déjà signalé les longues séries de livres de paye également par puits ou par atelier pour la SA des Mines de la Loire et Montrambert; pour la première, les données portent sur la période 1880-1930; elles remontent, pour la seconde, au commencement de la compagnie, mais ne dépassent pas les années 1920-1922 ; aucune de ces séries n'est complète.

Les archives des services financiers comportent des dossiers sur la gestion des actions des compagnies (RMF et Houillères de Saint- Étienne) et sur leurs portefeuilles dans la première moitié du XXe siècle (RMF, SA des Mines de la Loire, Montrambert) ; on trouve aussi un ensemble assez important sur les redevances tréfoncières pour la SA des Mines de la Loire. La relative richesse du fonds des Houillères de Saint-Étienne pour ce secteur tient à une série de dossiers fiscaux pour les années 30 et 40 et à des dossiers de règlements de créances à la suite d'une liquidation judiciaire.

Artificiellement gonflées pour la SA des Mines de la Loire et Montrambert par des séries de registres de pointage, aussi longues que celles des livres de paye et présentant des caractéristiques voisines, les archives du personnel n'en sont pas moins assez riches dans l'ensemble. On y trouve en particulier des règlements généraux et des dossiers sur l'organisation scientifique du travail (RMF et Montrambert), des registres d'entrée et de sortie et des données sur les mouvements du personnel, des dossiers sur la main d'œuvre étrangère et coloniale (ill.2), sur l'apprentissage (SA des Mines de la Loire) ou, ce qui est plus rare, sur les syndicats ouvriers. Les questions de sécurité y sont tout spécialement présentes, surtout dans les archives de Montrambert : désignation et activité des délégués mineurs, procès-verbaux d'accidents (depuis 1878 pour RMF, depuis 1890 pour Montrambert), dossiers sur des catastrophes (celle du puits Combes en 1928 pour RMF ; celles du puits de la Loire en 1939 et de La Chana en 1942 aux Mines de la Loire), mais aussi sur les maladies professionnelles et leur prévention (silicose et ankylostomiase).

Les archives des œuvres sociales apportent un éclairage complémentaire sur la gestion du personnel des houillères. Elles reflètent une politique paternaliste des compagnies qui, pour n'avoir pas été leur exclusivité (dans la région, la verrerie de Veauche ou les Établissements du Casino en offrent des exemples aussi remarquables), y ont été particulièrement, quoique inégalement, développées. Qu'elles occupent une place beaucoup plus grande dans les ar-

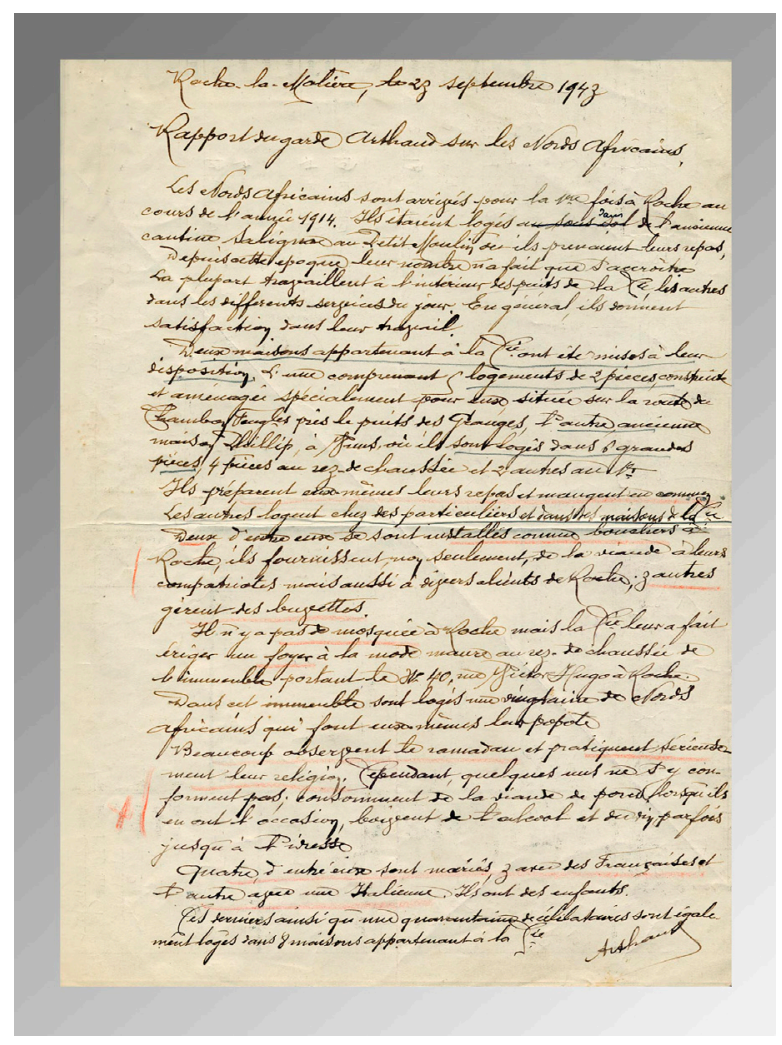

2. Rapport du garde Arthaud sur les Nords-Africains, Rochela-Molière, 23 septembre 1943 (20 × 25,7 cm., AD Loire, 1 ETP 510)

chives de certaines compagnies (Roche-la-Molière et Firminy, Montrambert) que dans d'autres (SA des Mines de la Loire, Houillères de Saint-Étienne) est sans doute révélateur ${ }^{7}$. Peut-être cette politique qui visait à stabiliser la main d'œuvre et tendait à accentuer le particularisme de la population minière trouvait-elle un terrain de développement plus favorable dans les petites villes nouvelles de la vallée de l'Ondaine où opéraient les premières que dans le cadre plus urbain de l'agglomération stéphanoise qui était le territoire des secondes. L'institution par vagues successives de ces œuvres, systématisées parfois en une « organisation sociale ॥ à l'époque de Vichy, ressort assez nettement des archives. Ce furent, d'abord, dès les années 1850, pour répondre à la grande dangerosité du métier, des établissements hospitaliers; puis la perspective devint plus familiale et, dans les années 1880, on créa des écoles pour les enfants des mineurs ; intervint ensuite, dans les années 1890, une pièce essentielle du dispositif : la construction de logements; enfin, après la guerre de 1914, on a assisté à un déploiement beau-

7 En revanche l'absence de dossiers dans les archives de la $C^{i e}$ des Mines de la Loire ne doit pas être interprétée trop hâtivement : c'est là en fait que cette politique a pris naissance. 


\section{Les sources de l'histoire minière aux Archives départementales de la Loire}

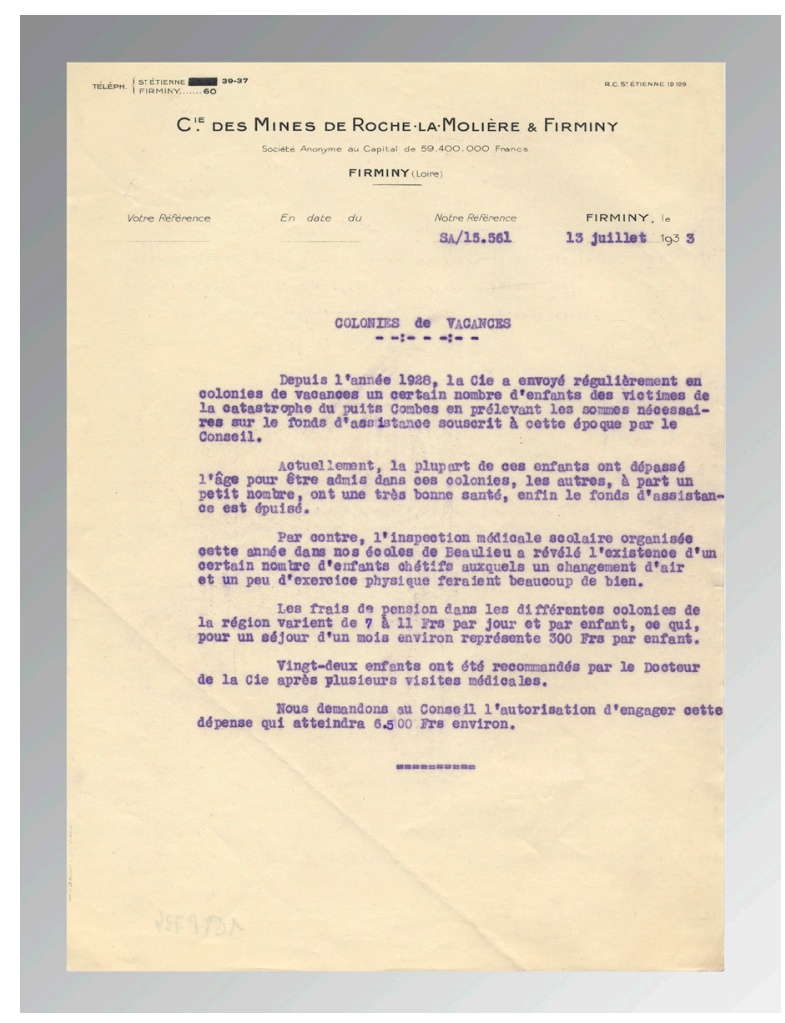

3. $C^{\text {ie }}$ des Mines de Roche-la-Molière et Firminy : note sur les colonies de vacances, Firminy, 13 juillet $1933(21 \times 27 \mathrm{~cm}$., AD Loire, 1 ETP 734)

coup plus diversifié : services sociaux, dispensaires, jardins ouvriers, colonies de vacances (ill.3), clubs sportifs, animations culturelles...

Avec les archives des domaines, on en arrive aux secteurs plus pauvres de ces fonds. Du moins pour celles versées au titre des Houillères du Bassin de la Loire. Étant donnée la continuité de gestion dans ce secteur, beaucoup de documents concernant les propriétés foncières et immobilières des Hovillères se retrouveront vraisemblablement dans les versements du service immobilier des Houillères de bassin du Centre et du Midi ${ }^{8}$. La plus grande ampleur de ce secteur dans les fonds de la $\mathrm{C}^{\text {ie }}$ de Roche-la-Molière et Firminy et de Montrambert et La Béraudière s'explique dans chacun des deux cas par des raisons différentes. Dans le premier, les dossiers sur les embranchements ferroviaires et les relations souvent difficiles entre la compagnie minière et celle du PLM occupent une place importante (ill.4). Dans le second, la masse principale est constituée par les archives d'un domaine forestier que la compagnie possédait en Sologne, et qui servait à son propre approvisionnement en boisages, mais aussi à celui d'autres compagnies minières dans

8 Voir ci-dessus note 6 le Nord (Anzin, Aniche), sans parler de la fabrication charbon de bois qu'on y pratiquait également...

Assez peu développées dans l'ensemble, les archives de l'exploitation proprement dite présentent d'une compagnie à l'autre un certain nombre de disparités. On trouve des dossiers sur l'attribution et la gestion des concessions et plus généralement sur les relations avec l'administration des mines, parfois des rapports périodiques des ingénieurs (SA des Mines de la Loire, et surtout, à partir de 1862, Montrambert), des séries de statistiques de production ou d'études de prix de revient, de rares journaux de mines (Montrambert), des rapports techniques (RMF, Cie des Mines de la Loire ou Montrambert), un seul dossier sur les brevets d'invention (RMF, à partir de 1929), quelques-uns sur le matériel d'exploitation (portant sur les années 19201940). Aspect pourtant essentiel, la transformation du charbon n'est représentée que par un dossier unique sur les fours à coke de la $\mathrm{C}^{\mathrm{ie}}$ de Montrambert.

Encore moins fournies sont les archives commerciales, et aucune compagnie ne fait exception cette fois. On peut quand même signaler un registre des acheteurs (pour RMF, à partir de 1935), des états ou tableaux de ventes en série discontinue (1901-1919 et les années 30, pour les Mines de la Loire), quelques dossiers de correspondance commerciale, concernant des fournitures de charbon à la Marine nationale (dans la deuxième moitié du XIXe siècle), des fournitures de gaz et d'électricité à différentes entreprises, des exportations en Belgique ou en Italie.

\section{Les archives de l'administration des mines}

Pour la période allant de 1800 à 1940, ces archives font réglementairement partie de la sous-série $8 \mathrm{~S}$; pour la période postérieure, il n'y a eu à ce jour qu'un seul versement de la Direction régionale Industrie Recherche Environnement (DRIRE) (2065 W), organisme où restent encore conservés les dossiers des concessions non encore liquidées. Quant aux dossiers de la série S, ils ne font l'objet pour l'instant que d'un classement provisoire très insatisfaisant ; leur classement définitif devrait intervenir pendant l'année 2008-2009. C'est donc un ensemble qui reste aujourd'hui assez mal connu, et nous nous bornerons à en indiquer de façon très sommaire deux grandes lignes de force.

La première concerne les différents aspects de la mise en œuvre de la loi de 1810. II faut citer d'abord les documents assez exceptionnels que représentent les plans dessinés par l'ingénieur en chef Louis-Antoine Beaunier en 1812 pour préparer la délimitation des concessions à attribuer : un peu plus de soixante 

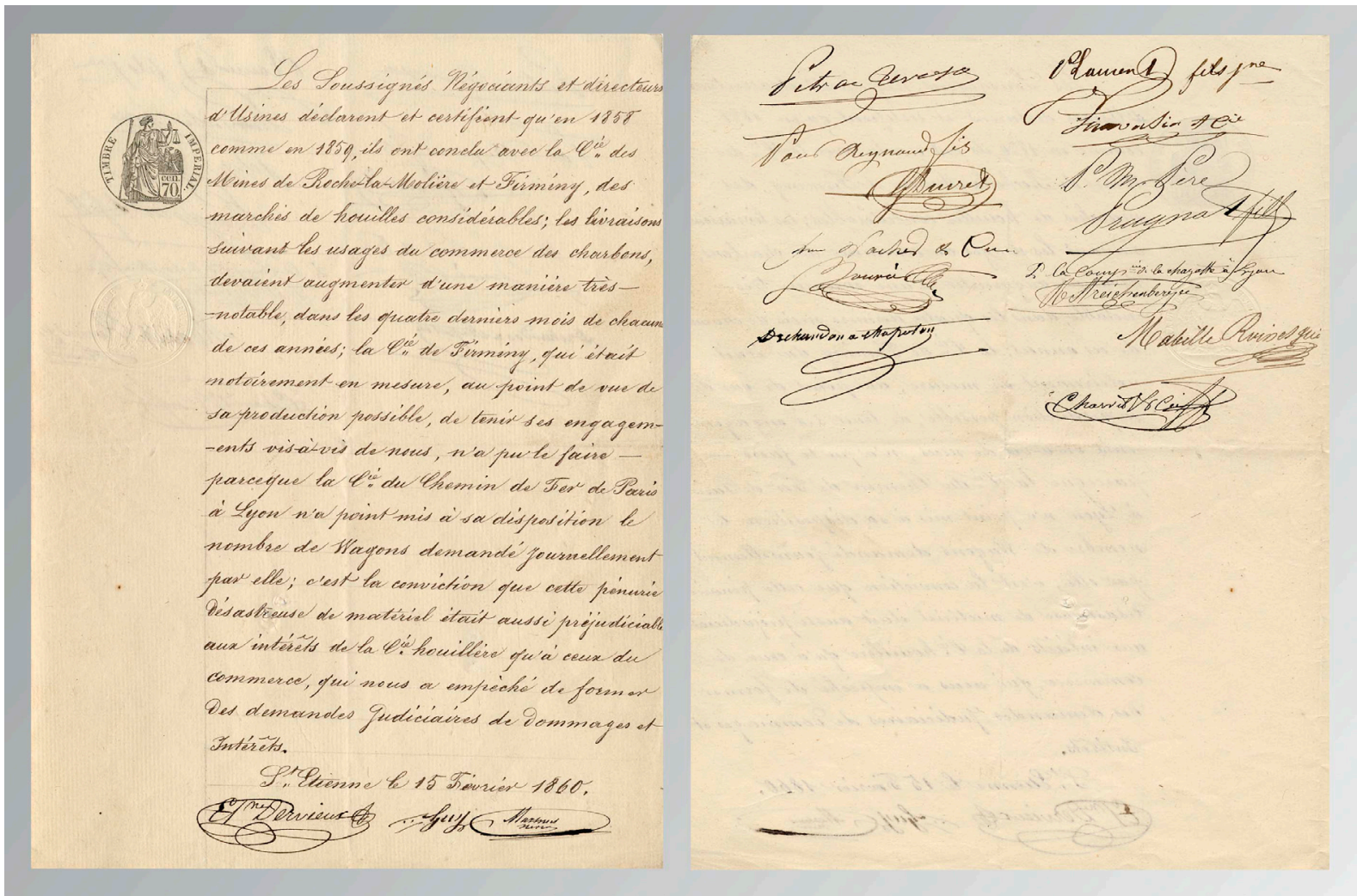

4. Déclaration de négociants et directeurs d'usines en faveur de la $\mathrm{C}^{\text {ie }}$ des Mines de Roche-la-Molière et Firminy, SaintÉtienne, 15 février 1860 (17,5 × 25 cm., AD Loire, 1 ETP 779)

planches aquarellées représentant le territoire minier sous tous ses aspects (ill.5), et qui n'ont été versées aux Archives départementales par la DRIRE qu'en décembre 2000\%. On trouvera là aussi évidemment les dossiers d'attribution des concessions et tout ce qui concerne le contrôle permanent de l'exploitation par les ingénieurs du Corps des mines : des enquêtes publiques par exemple lancées en vue de l'autorisation de certains travaux miniers ou, au contraire, à propos de la fermeture de tel ou tel site d'extraction (ill.6).

La Loire a connu entre 1876 et 1891, une longue série de catastrophes dues à l'explosion du grisou (puits Jabin en février 1876, puits Chatelus et Montmartre en mars 1887, puits Verpilleux, Saint-Louis, Mars et Jabin en juillet 1889, puits Pélissier en juillet 1890, puits Ambroise en août 1890 et puits de La Manufacture en décembre 1891). Ces accidents ont été pour beaucoup dans l'adoption d'une autre loi importante, celle du 8 juillet 1890, instituant des délégués mineurs, chargés du contrôle des conditions de sécurité au fond. Toute une partie des dossiers de la sous-série $8 \mathrm{~S}$ concer-

9 Ces documents ont été numérisés. nent l'application de cette loi : dossiers d'organisation des élections de délégués, séries de rapports sur les visites qu'ils faisaient au fond ... Plus généralement, on trouvera une importante documentation sur les accidents du travail et les conditions de sécurité, qui complète celle des compagnies à ce sujet. De façon significative, 28 des 37 dossiers de l'unique versement de la DRIRE portent sur les accidents du travail dans les mines depuis 1940 jusqu'à la fermeture.

\section{Aperçus sur les sources complémentaires}

En dehors de cette sous-série $8 \mathrm{~S}$ dont une grande partie concerne spécifiquement l'histoire de l'exploitation minière, on trouve une documentation parfois assez riche dans d'autres séries d'archives publiques. Il s'agit en particulier de la série C (administrations provinciales d'Ancien régime), de la série $L$ (période révolutionnaire), de la série $M$ (administration générale et économie, 1800-1940) et des versements du cabinet du préfet après 1940 (série W), et de façon plus accessoire des séries $R$ (affaires militaires), $U$ (justice) ou X (assistance et prévoyance). Les archives de deux établissements publics intéressent aussi l'histoire minière : celles de la Chambre de commerce et d'industrie de Saint-Étienne (formant provisoirement la sous-série $13 \mathrm{~J}$ en attendant un reclassement définitif sous la cote 5 ETP) et celles de l'École nationale des 


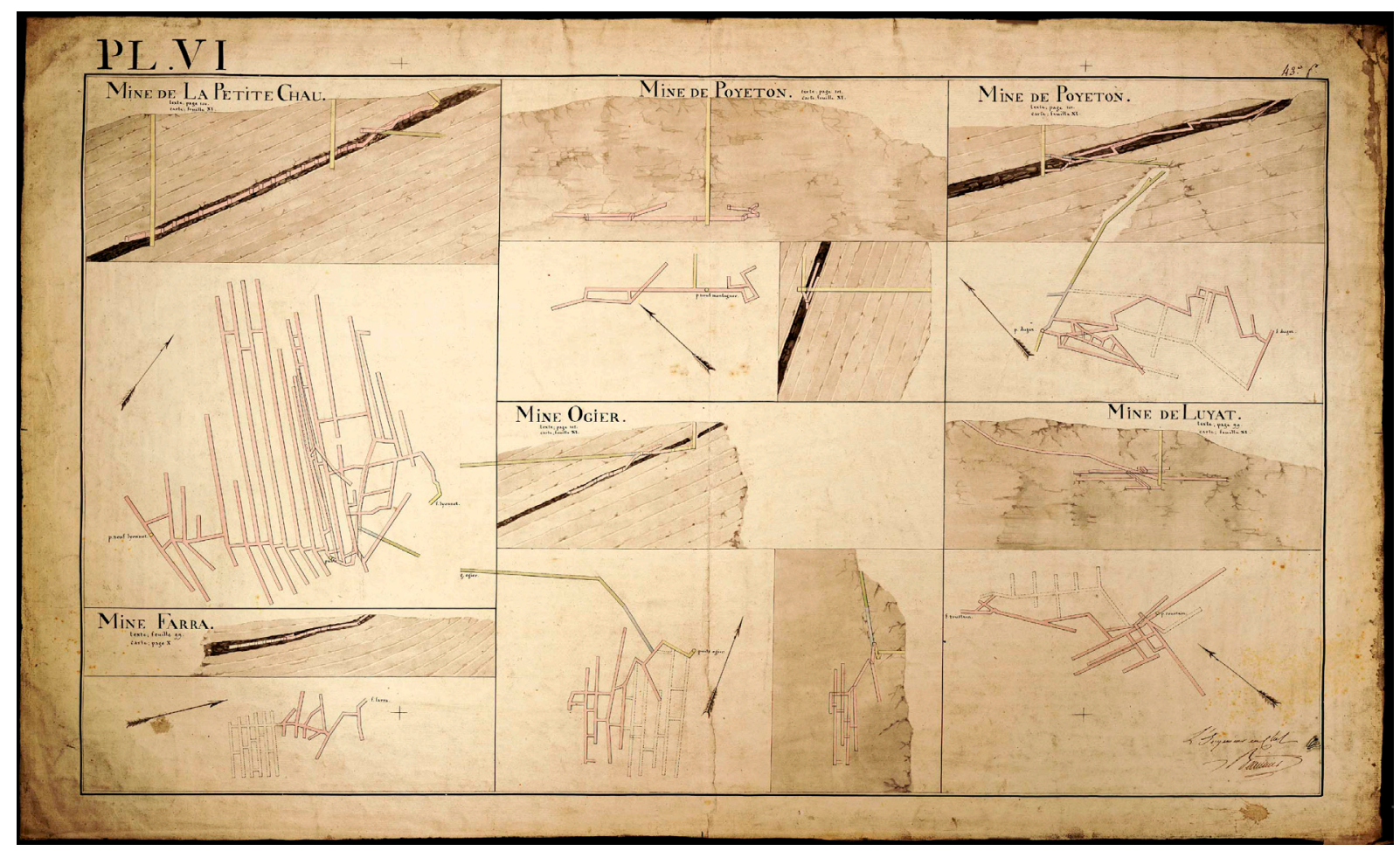

5. Atlas de Louis Beaunier (planche VI) [1812] (101 x 64 cm., AD Loire, S non coté)

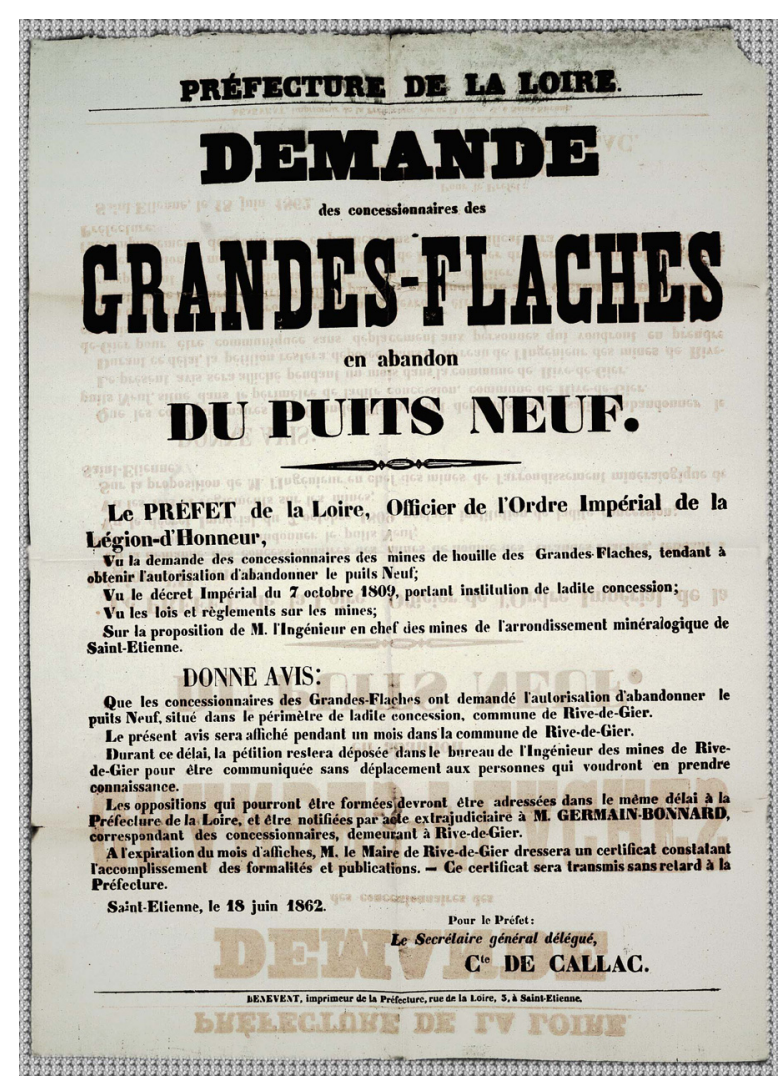

6. Demande des concessionnaires des Grandes Flaches en abandon du puits Neuf, affiche, Saint-Étienne, 18 juin 1862 (48 x 65,5 cm., AD Loire, cote provisoire $S 1388)$
Mines de Saint-Étienne (aujourd'hui $106 \mathrm{~J}$, et devant constituer ultérieurement la sous-série 6 ETP) ${ }^{10}$. E† puis on trouvera des ressources parfois très précieuses dans certains fonds d'archives privées. II s'agit pour quelques uns d' archives d'entreprises minières proprement dites, comme celles de la Compagnie des anthracites de Bully - des registres de copies de lettres entre 1917 et 1921 (1 J 421) -, ou celles incluses dans les papiers de Thomas Hutter, qui fut entre autres choses un acteur de cette histoire (45 J) : archives concernant plusieurs petites exploitations dans les années 1830-1840, archives de la petite compagnie des mines du Couloux entre 1814 et 1878, archives aussi provenant de la Compagnie des mines de la Loire (où Thomas Hutter a exercé différentes responsabilités) et de la Compagnie de Montrambert et de La Béraudière (dont il a été directeur entre 1854 et 1878). Le fonds de la famille Husson (125 J) est composé des papiers de deux ancêtres de cette famille : Maximilien Evrard qui fut directeur des Mines de la Chazotte entre 1852 et1872, et Léon Michel, chef du contentieux des Houillères de Saint-Étienne dans les années 1870-1880. On trouve également quelques éléments sur les exploitations du Cros et de Monthieu (à Saint-Étienne) au $X I X$ siècle dans les archives de la famille Bernou de Rochetaillée (157 J). Un long contentieux sur des droits tréfonciers dans les années 1920 a donné lieu au ras-

10 L'École nationale des mines avait également constitué une très importante bibliothèque dont le fonds ancien a été déposé à la Médiathèque municipale de Saint-Étienne. 


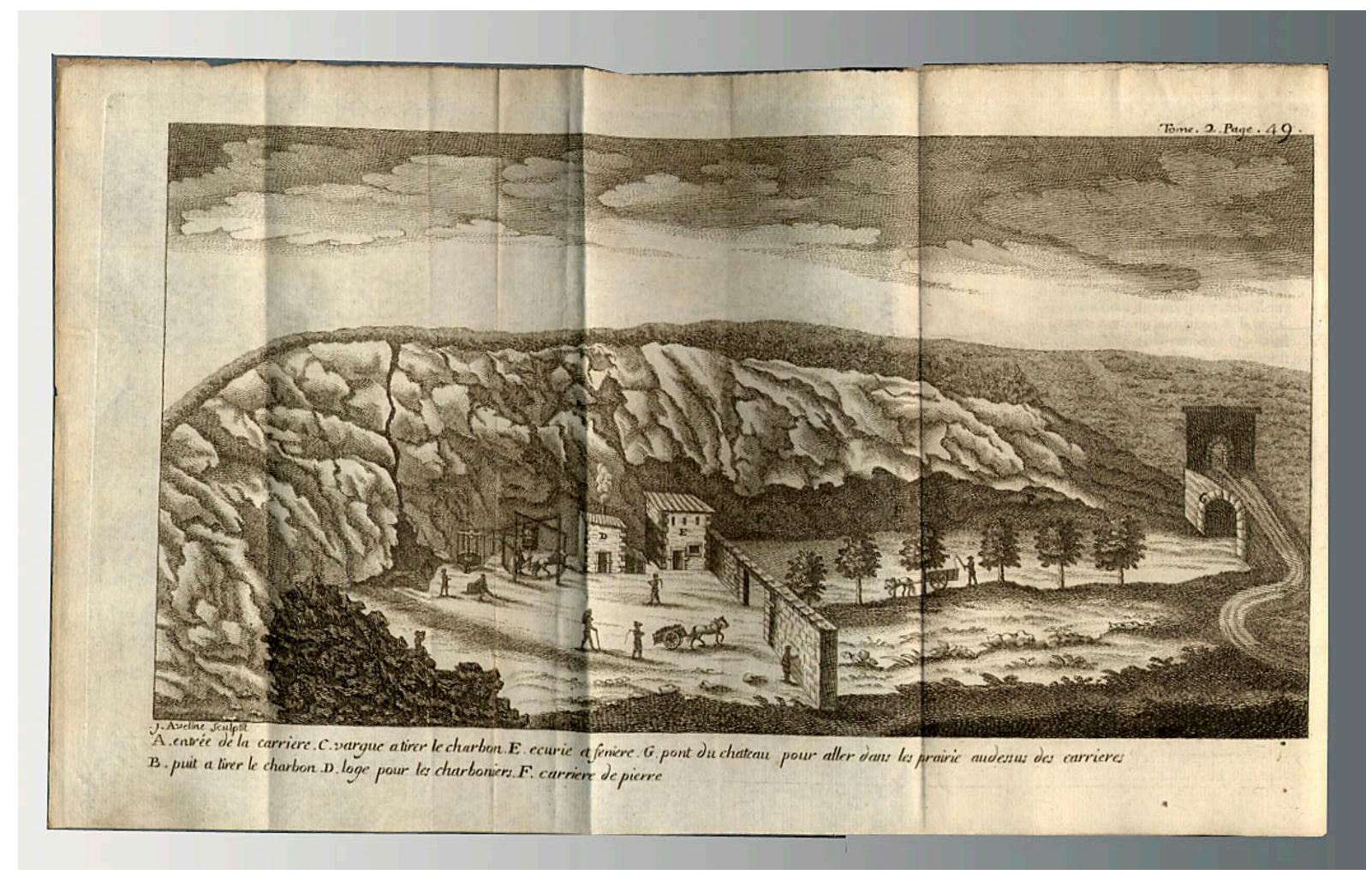

7. Gravure de J. Aveline représentant une exploitation de charbon en carrière (illustration de l'«Histoire naturelle et générale du charbon minéral ", dans Alléon-Dulac, Mémoires pour servir à l'histoire naturelle des provinces de Lyonnois, Forez et Beaujolois, t.Il, Lyon, Claude Cizeron, 1775) (32 × 17,5 cm., AD Loire, Chaleyer 4787)

semblement d'une documentation très riche sur l'exploitation dans la partie orientale du bassin depuis les premières décennies du XIX siècle (fonds BertholonThévenet, $178 \mathrm{~J}$ ). D'autres fonds concernent plus indirectement l'histoire minière, comme, nous le verrons, celui de la Compagnie du canal de Givors (1 19 J). E† il ne faudrait pas omettre l'apport des imprimés : des ouvrages statistiques sur le département des XVIII et XIXe siècles par exemple, ou bien le journal Le Rhône et Loire, publié par la $C^{i e}$ des Mines de la Loire entre octobre 1847 et février 1848 ; nous en donnerons plus bas quelques autres exemples. Enfin, on n'oubliera pas non plus l'apport de l'iconographie : les cartes postales représentant les installations de surface ou quelques aspects du travail au fonds (sous-série $2 \mathrm{Fi}$ ), un ensemble de photographies donné récemment par les héritiers d'un ancien cadre des HBL, Marius Chalendard (sous-série 19 Fi) ${ }^{11}$.

11 Pour être tout à fait complet, il faudrait évoquer aussi les archives concernant les mines de la Loire conservées dans d'autres dépôts. Comme, aux Archives nationales, celles du
D'un point de vue thématique, ces sources complémentaires présentent quelques lignes de force que nous allons passer brièvement en revue. II s'agira d'abord de certains moments saillants de l'histoire de l'exploitation dans son ensemble; puis nous évoquerons les apports de ces différents fonds sous trois angles plus particuliers : les aspects technique, économique et humain.

Cinq moments de l'histoire minière peuvent être plus spécialement mis en lumière. C'est d'abord l'histoire précoce de l'exploitation dont on trouvera trace, dans quelques ouvrages imprimés (ill.7) et dans quelques dossiers des séries $C$ et $L$, avec des états d'extraction et des documents concernant l'attribution des premières concessions ${ }^{12}$. Un "état général des mines de Rive-de-Gier $॥$, conservé dans les archives de la Société des sciences de Rive-de-Gier (2 J 13), offre un tableau d'ensemble très précis de la situation en l'an II dans ce secteur où, rappelons-le, l'exploitation a été le plus anciennement développée. Certaines des cartes qui ont été établies à l'occasion du contentieux tréfoncier Bertholon-Thévenet (178 J) donnent une bonne image du morcellement qui a

Conseil des Mines. Nous en citerons plus bas (notes 12 et 17) deux autres exemples.

12 Cotes C 78, 83/1, 86 et 90 ; L 818-828. On trouvera sans doute davantage d'éléments encore dans la série $C$ des archives du Rhône, puisque l'actuel département de la Loire faisait partie de la généralité de Lyon. 


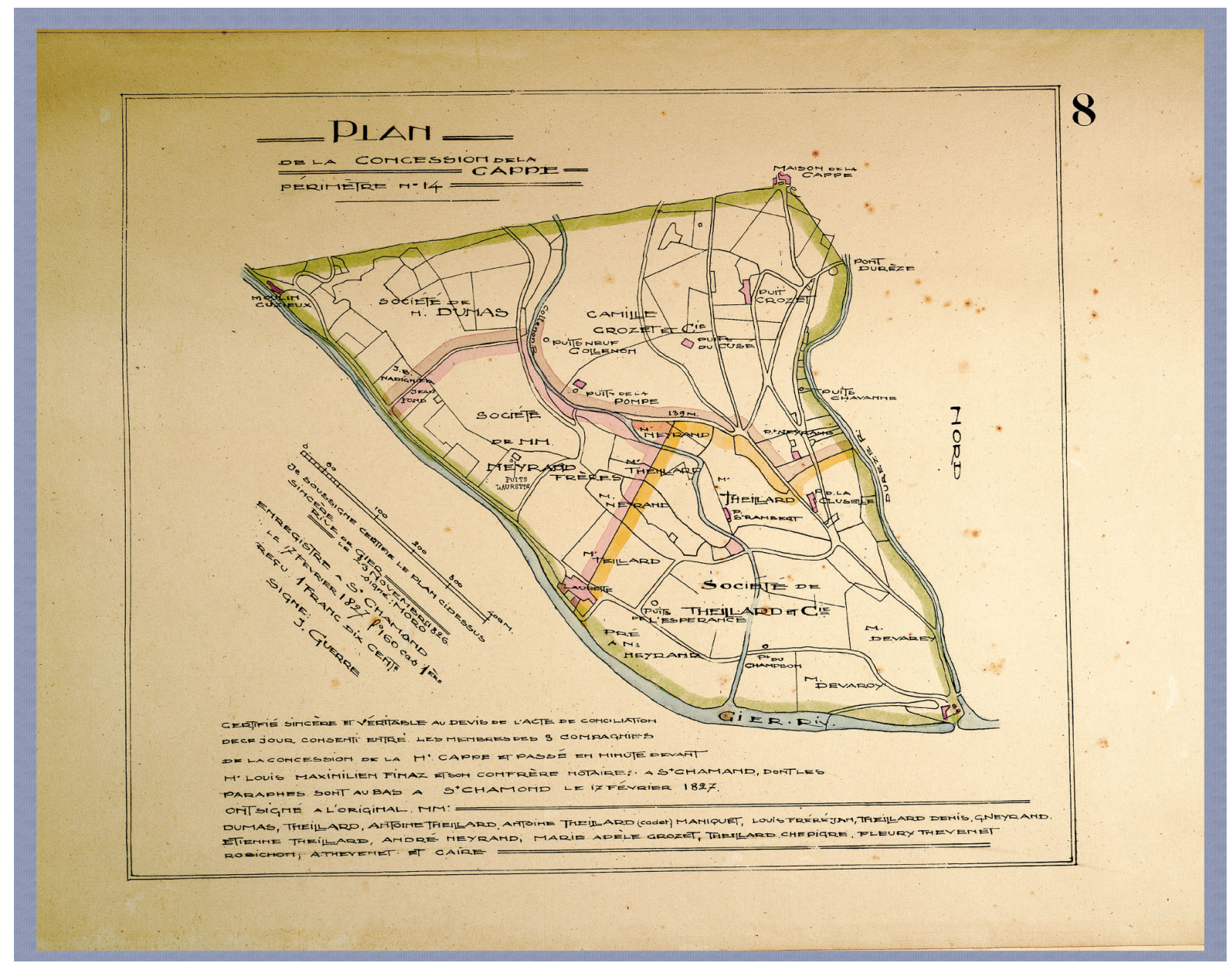

8. Plan de la concession de la Cappe en 1826, [copie circ. 1913] (55 x 38 cm., AD Loire, 178 J 33)

persisté dans cette zone jusqu'aux années 1830 (ill.8), où s'amorce le deuxième moment significatif, celui de la concentration. Sur ce processus, les papiers de Thomas Hutter apportent un matériau très riche, qui pourrait être complété par les minutes des notaires de Rive-de-Gier. Hutter était lui-même alors notaire et il a participé activement, à ce titre, au transfert de la propriété des exploitations des mains de notables ou de petits extracteurs locaux à celles de groupes de capitalistes lyonnais (ill.9), transfert qui a préparé et accompagné la formation de la grande Compagnie des mines de la Loire ${ }^{13}$. On trouvera aussi dans

13 En particulier 45 J 96-122. Voir, pour quelques précisions supplémentaires sur ce point, Denys Barau, « Trois pistes de recherches à partir des fonds industriels des Archives départementales de la Loire "), dans Didier Nourrisson, Jean-François Brun, Marie-Thérèse Avon-Soletti, Bernard Bacher éd., Histoire contemporaine et patrimoine : la Loire, un département en quête de son identité,Saint-Étienne, Publications de l'université de Saint-Étienne, 2008. On pourra consulter aussi le catalogue d'exposition Thomas Hutter, Rive-de-Gier, 1830-1852, le dossier 8 M 69 des données sur le point de vue des autorités concernant ce processus de concentration. Quant à la Compagnie qui en a été le résultat, la vive polémique qu'elle a fait naître s'est manifestée en une série de brochures, qui sont une autre source encore. Dans les dernières décennies du siècle, c'est le troisième moment, certaines petites exploitations qui avaient survécu à la concentration furent abandonnées par des propriétaires qui les jugeaient désormais trop peu rentables; deux d'entre elles, à Rivede-Gier et à Monthieu (Saint-Étienne), furent reprises par leurs ouvriers qui menèrent pendant une vingtaine d'années une expérience de coopérative connue sous le nom de "mine aux mineurs ") deux dossiers de la série M lui sont consacrés (10 M 503 et 504) 14. $^{14}$. Quatrième moment significatif, plus important, celui de la seconde guerre mondiale, avec la séquence collaboration-épuration-nationalisation : on trouvera sur cette phase là un complément aux documents internes de la sous-série 1 ETP dans les dossiers de la

Saint-Étienne, Archives départementales de la Loire, 1991.

14 Ce mode de gestion a connu un plus grand succès dans l'industrie verrière, où on a compté six coopératives; une d'entre elles au moins fonctionnait encore en 1940. 


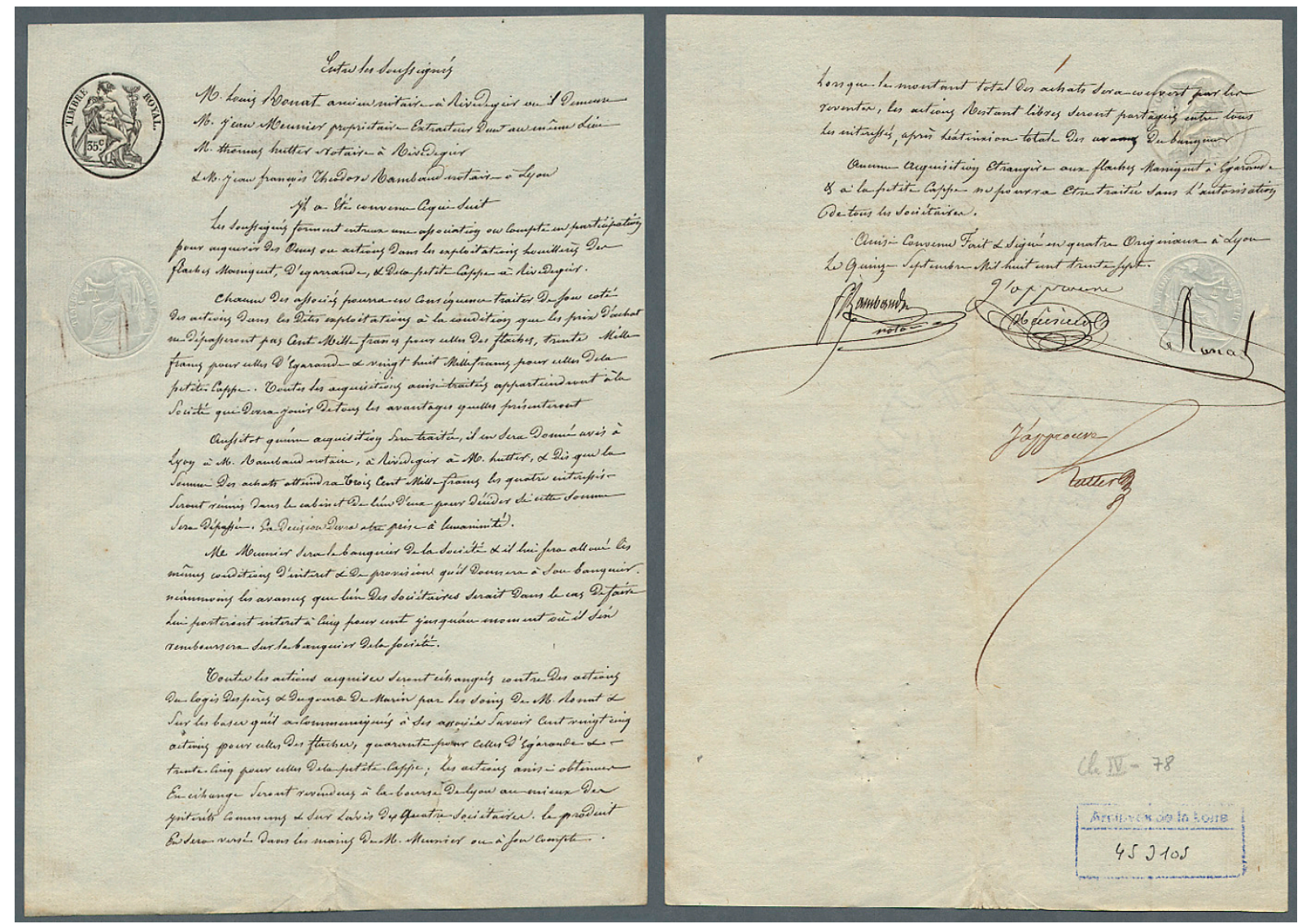

9. Convention pour l'achat d'onces d'exploitations hovillères, Lyon, 15 septembre 1837 (17,7 × 24,7 cm., AD Loire, 45 J 105)

série W provenant du cabinet du préfet ( $85 \mathrm{~W} 137$ pour l'épuration ; 97 W 12 pour la nationalisation) ainsi que dans la presse locale. De même pour le cinquième de ces moments, le moment de la fermeture, prévue, redoutée, reportée et finalement accomplie dans les années 1970 et 1980.

Sur l'aspect technique de l'exploitation, la masse documentaire la plus importante est constituée par les dossiers d'établissements classés de la sous-série $5 \mathrm{M}$. Elle ne porte pas seulement sur les installations d'extraction, mais aussi sur celles qui servaient aux différentes transformations de la houille, sur lesquelles nous avons vu que les archives des compagnies étaient des plus lacunaires : cokeries, fabriques d'agglomérés, productions de goudrons, usines à gaz... ${ }^{15}$. On trouvera aussi des études à caractère technique dans les archives de la Chambre de commerce de Saint-Étienne, comme, par exemple, une étude rédigée en 1892 sur l'électrification (13 J 21). Certains contentieux, comme les dégâts de surface ont pu aussi donner lieu à la production d'archives judiciaires, comme les rapports

15 Cotes 5 M 419-456. d'experts, riches en information de caractère technique (série U). S'agissant par ailleurs des apparences extérieures de l'exploitation, de la manière dont elle s'inscrivait dans le paysage, l'iconographie offre un secours précieux : aux très nombreuses cartes postales (sous-série $2 \mathrm{Fi}$ ) représentant les chevalements et les installations de surface, surtout dans la période 1900-1914 (ill.10), on pourra comparer une série des photographies des puits les plus récents, prises dans les années 1950 (sous-série 19 Fi).

Du point de vue économique, nos sources complémentaires apportent surtout des éléments sur la question des débouchés, qui ne fait pas l'objet d'archives spécifiques très nombreuses dans les fonds des compagnies ${ }^{16}$. Les archives de la Chambre de commerce apporteront des éléments plus généraux en particulier par rapport aux tarifs douaniers et aux traités de commerce internationaux : les réactions par

16 L'exploration des archives des organismes professionnels et des filiales communes aux compagnies permettra peutêtre de combler en partie cette lacune à l'avenir. Des développements sont consacrés à cette question dans le livre de Michel Cotte, Le choix de la révolution industrielle : les entreprises de Marc Seguin et de ses frères, 1815-1835, Rennes, Presses universitaires de Rennes, 2007. 


\section{Les sources de l'histoire minière aux Archives départementales de la Loire}

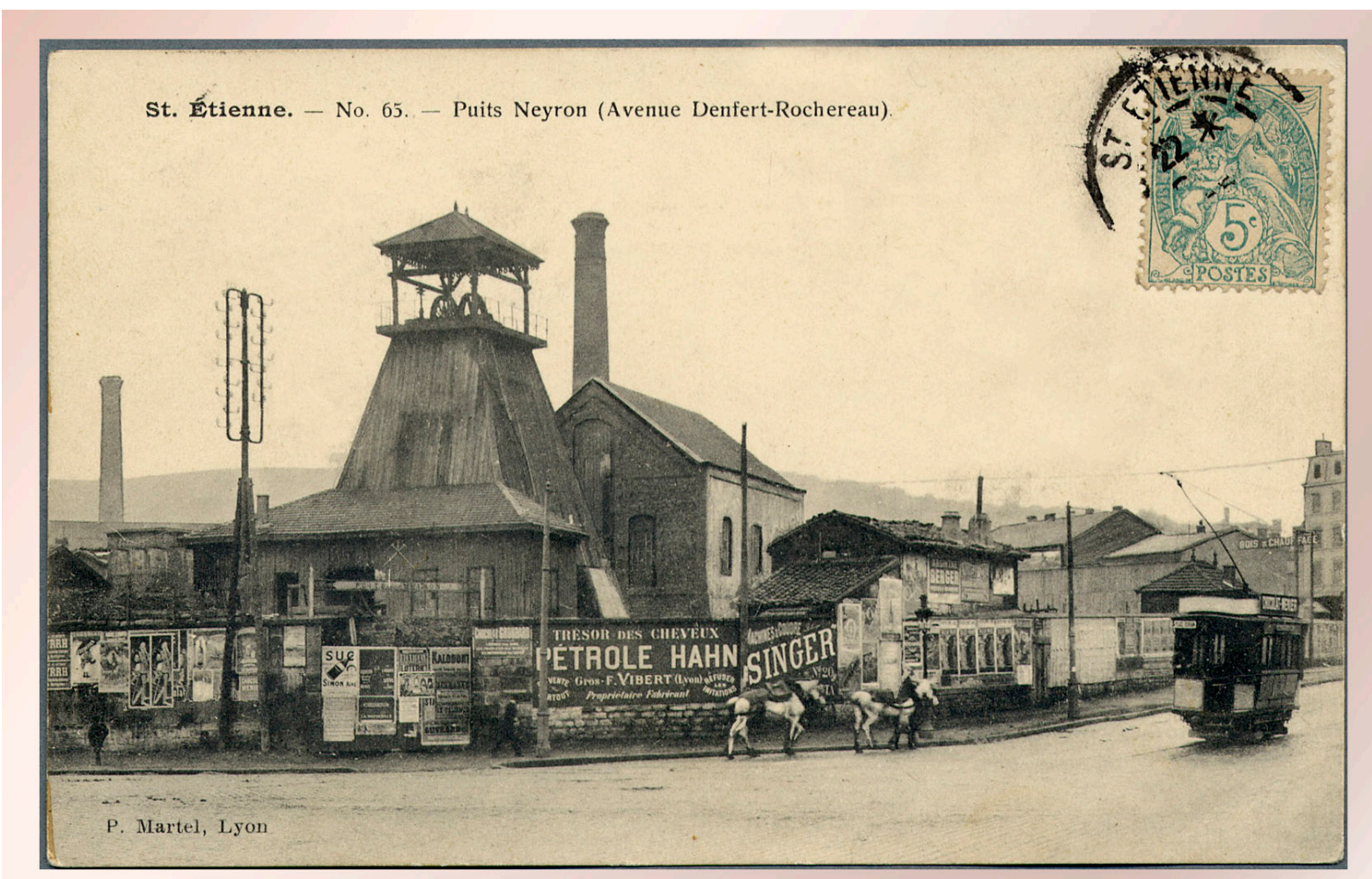

10. Puits Neyron (Saint-Étienne), carte postale P. Martel, Lyon, [ circ.1900 ] (14 × 9 cm, A D Loire, 2 Fi 2221)

exemple de la profession au traité de libre-échange avec l'Angleterre de 1860. Sous une toute autre forme, on aura des données quantitatives assez intéressantes sur la destination du charbon stéphanois dans les archives de la Compagnie du Canal de Givors (1 $19 \mathrm{~J}$ ) qui assurait dans la première moitié du XIXe siècle le transport du charbon de la partie occidentale du bassin jusqu'au Rhône, et de là à la région lyonnaise et surtout à la France du midi. On pourra consulter aussi la sous-série 3 S (voies navigables) au sujet de l'activité de ce même canal et de la navigation sur la Loire et sur le canal de Roanne à Digoin qui a assuré en partie l'exportation du charbon vers la région parisienne et la France du nord. Sur ce point, on trouvera d'autres éléments dans la sous-série $5 \mathrm{~S}$ consacrée aux chemins de fer: on s'en souvient, la première ligne construite en France, entre Saint-Étienne et Andrézieux, était destinée au transport du charbon jusqu' à la Loire (ill. 11 1) 17.

17 II est du reste à noter que la famille de Marc Seguin, qui a été à l'origine de la deuxième ligne de chemin de fer française, la ligne Saint-Étienne-Lyon, avait des intérêts dans les mines de la Loire. Les très riches archives de cette famille conservées aux Archives départementales de l'Ardèche (sous-série $41 \mathrm{~J})$ en portent trace. Michel Cotte, qui a fondé l'ouvrage
Un prospectus édité sans doute dans les années 1950 par les HBL témoigne, en même temps que de l'empire grandissant de la publicité, de la volonté de diversifier les débouchés (1 J 801, ill.12).

Incontestablement, la documentation complémentaire la plus riche porte sur la dimension humaine de l'exploitation. L'encadrement d'abord et le monde des ingénieurs des mines, qu'on retrouve aussi bien au sein des compagnies, surtout après qu'elles eurent acquis une certaine ampleur, que dans l'administration des Mines, avec les archives de l'École des mineurs, devenue plus tard l'École nationale des Mines de Saint-Étienne, créée par l'ordonnance du 2 août 1816 à l'instigation de Louis Beaunier. Les archives propres de cette institution peuvent être complétées par une série de dossiers de la série $M^{18}$. Mais c'est avant tout sur le personnel ouvrier que la documentation est abondante. On pense d'abord à l'aspect revendicatif, à la place éminente de la corporation minière dans le mouvement ouvrier : son organisation syndicale fait l'objet de six articles de la sous-série 10 M (10 M 464469) ; vingt-trois autres (10 M 317-339) concernent les

cité dans la note précédente sur l'étude de ces sources, en a donné une présentation très détaillée sous le titre Le fonds d'archives Seguin : aux origines de la révolution industrielle en France, 1790-1860, Privas, Archives départementales de l'Ardèche, 1997.

18 Cotes 9 M 212-220. 


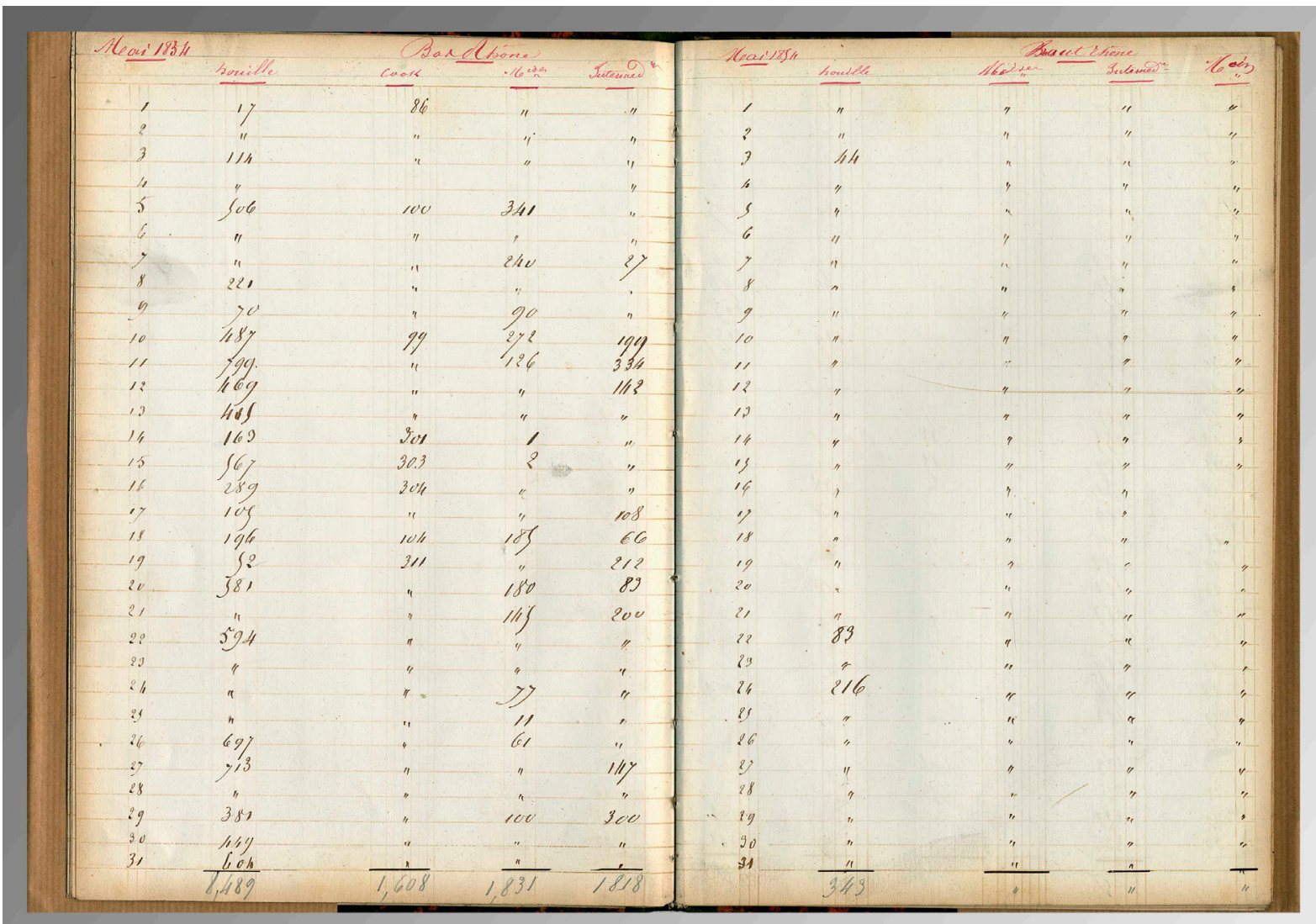

11. Cie du canal de Givors : registre des destinations des marchandises, février 1854 (39,6 × 28,7 cm., AD Loire, 119 J 276)
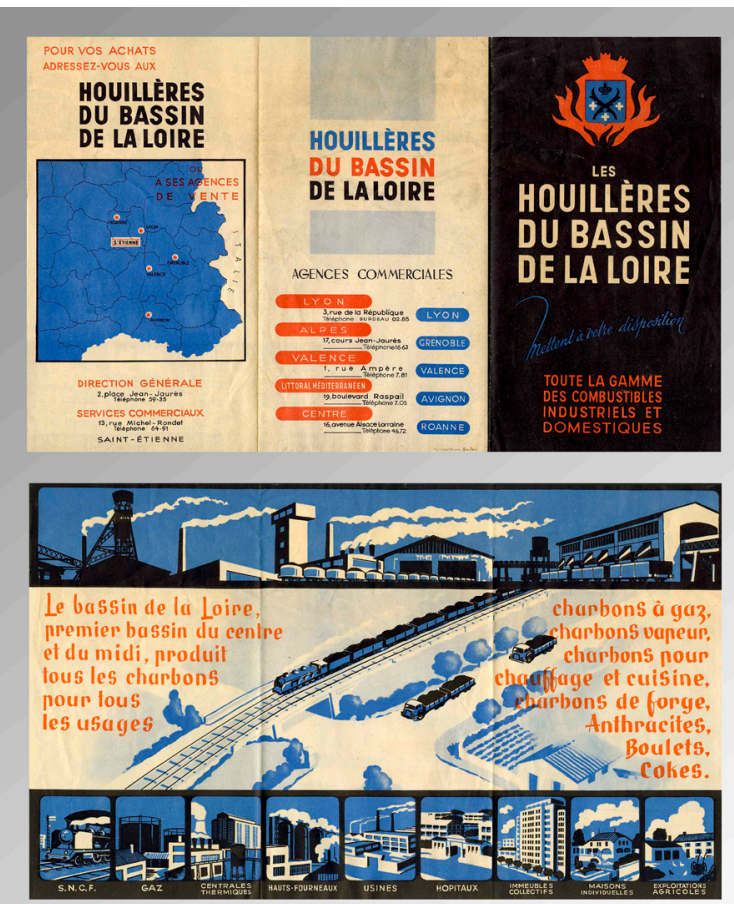

12. Houillères du bassin de la Loire : dépliant publicitaire, [années 1950] (3 × 14,2 × 29,6 cm., AD Loire, 1 J 801) très nombreuses grèves qui ont affecté tout ou partie du bassin depuis 1830 jusqu'à la Seconde Guerre mondiale; on ne compte pas moins de cinq dossiers dans les archives du cabinet du préfet (85 W 201-205) sur la grande grève d'octobre-décembre 1948. Les archives des unions départementales de la CGT-FO et de la CFTC-CFDT livrent le point de vue syndical : ce sont dans les premières les comptes-rendus des réunions du comité fédéral des mineurs de la CGT pour la période 1933-1938; dans les secondes, des dossiers sur l'activité des syndicats de mineurs entre 1948 et 1962. On possède aussi, grâce à quatre photographies sur plaque de verre (19 Fi), des images du célèbre épisode de la fusillade du Brûlé à La Ricamarie en juin 1869 (ill.13). Mais d'autres aspects moins spectaculaires de la vie des ouvriers mineurs sont également concernés. Les sociétés de secours mutuels ont été très développées dans cette branche - la dureté particulière du métier l'explique aisément -, on en trouvera trace dans la série $X$; à cause du rôle stratégique des houillères, les mineurs ont été soumis à réquisition en temps de guerre: on peut en suivre la mise en œuvre dans l'article 2 R 174 ; l'important fonds de la police des étrangers (4 M 436-537, 1018-1019, 10211140 ) permettrait d'étudier de plus près le recours à la main d'œuvre immigrée. La place de la figure du mineur dans l'imaginaire social est enfin inséparable des 


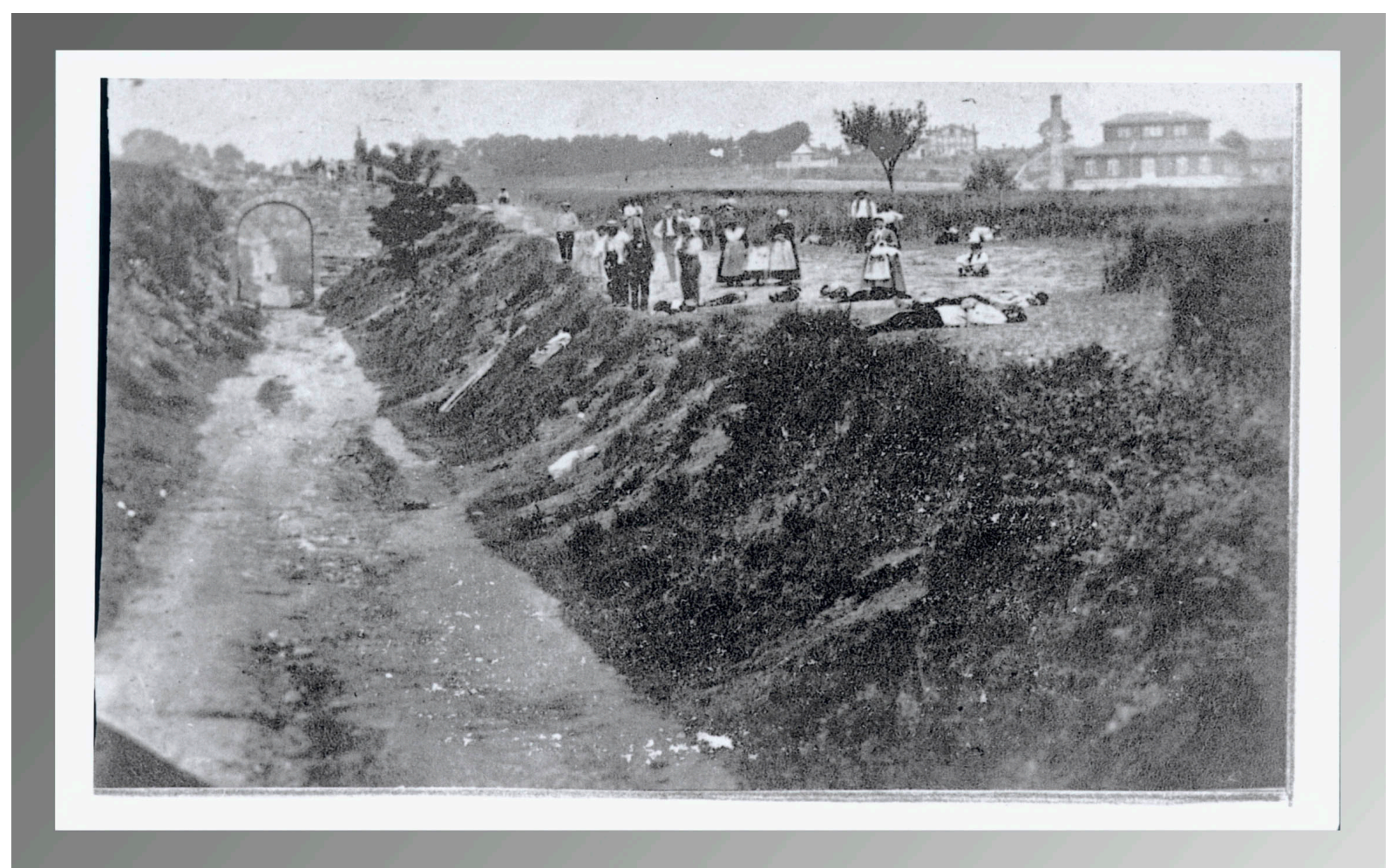

13. Épisode des incidents du Brûlé, 16 juin 1869, (tirage à partir d'une photographie sur plaque de verre), (19,5 x $14 \mathrm{~cm}$., AD Loire, 19 Fi non coté)

dangers encourus auxquels elle a emprunté un certain ton d'épopée ; il n'a jamais été aussi sensible qu'au moment des catastrophes qui ont endevillé périodiquement le bassin de la Loire comme tous les autres : les dossiers conservés à ce sujet dans la série M ( 1 M 744-753) reflètent l'émotion très largement ressentie et des mouvements de solidarité qui ont largement dépassé le cadre local.

Au fil de cet inventaire un peu rapide, nous avons voulu donner une idée d'une documentation abondante et bien diversifiée. Elle n'a été jusqu'à présent qu'assez peu exploitée. Depuis l'ouvrage de LouisJoseph Gras, qui reste un référence indispensable à cause de sa très riche information, mais participe d'un style d'historiographie aujourd'hui tout à fait dépassé ${ }^{19}$, les études consacrées à la mine dans le bassin stéphanois n'ont pas été très nombreuses. On peut citer, outre le livre important de Pierre Guillaume sur la Compagnie des Mines de la Loire ${ }^{20}$, les travaux de

19 Louis-Joseph Gras, Histoire économique générale des mines de la Loire, Saint-Étienne, Impr. Théolier, 1922.

20 Pierre Guillaume, La Compagnie des mines de la Loire, 1846-1854 : essai sur l'apparition de la grande industrie capitaliste en France, Paris, Presses universitaires de France, 1966.
Maurice Bedoin sur l'archéologie minière ${ }^{21}$, la monographie de Jean-Paul Burdy sur un quartier de mineurs de Saint-Étienne, le quartier du Soleil 22, les ouvrages de Thierry Veyron et de Luc Rojas sur I'histoire technique ${ }^{23}$, et le livre d'Anne-Françoise Garçon sur l'École des mines ${ }^{24}$. Peut-être le classement prochain de la sous-série $8 \mathrm{~S}$ et l'achèvement de celui des archives des Hovillères du bassin de la Loire permettront-ils un dévelopement plus actif de la recherche dans ce domaine.

21 Maurice Bedoin, Le Patrimoine minier stéphanois, Rochela-Molière, édité par l'auteur, 1981.

22 Jean-Paul Burdy, Le Soleil noir : un quartier de Saint-Étienne, 1840-1940, Lyon, Presses universitaires de Lyon, 1989.

23 Thierry Veyron, Essai sur la mécanisation du fond dans les houillères, Saint-Étienne, Publications de l'université de SaintÉtienne, 1998, et Essai sur l'évolution technique des houillères françaises et belge, 1800-1880, Paris, L'Harmattan, 1999. LuC Rojas, Histoire de révolution technologique : de l'infrastructure artisanale à la grande industrie hovillère de la Loire, Paris, L'Harmattan, 2008.

24 Anne-Françoise Garçon, Entre l'État et l'usine : l'École des Mines de Saint-Étienne au XIXe siècle, Rennes, Presses universitaires de Rennes, 2004. 


\section{TABLEAU COMPARATIF DES FONDS DES DIFFERENTES COMPAGNIES}

\begin{tabular}{|c|c|c|c|c|c|c|}
\hline & $\begin{array}{c}\text { Cie }^{\text {de Roche-la- }} \\
\text { Molière et Fir- } \\
\text { miny }\end{array}$ & $\begin{array}{c}C^{\mathrm{ie}} \text { Mines de la } \\
\text { Loire }\end{array}$ & $\begin{array}{l}\text { SA des Mines de } \\
\text { la Loire }\end{array}$ & $\begin{array}{l}\text { Houillères de } \\
\text { Saint-Étienne }\end{array}$ & $\begin{array}{c}\mathrm{C}^{\mathrm{ie}} \text { de Montram- } \\
\text { bert et de } \\
\text { La Béraudière }\end{array}$ \\
\hline 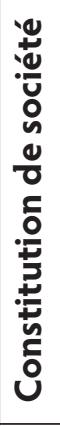 & & $\begin{array}{l}\text { Statuts } \\
(1811-1943) \\
\text { État des action- } \\
\text { naires } \\
\text { (1910-1919) } \\
\text { Dossier de } \\
\text { nationalisation } \\
\text { (1945) } \\
\text { Historique } \\
\text { (8 articles) }\end{array}$ & $\begin{array}{l}\text { Statuts } \\
(1844-1857) \\
\text { Contestation du } \\
\text { monopole } \\
(1845-1854) \\
\text { Fractionnement } \\
(1850-1858) \\
\\
\text { (9 articles) }\end{array}$ & $\begin{array}{l}\text { Statuts } \\
(1894-1941)\end{array}$ & $\begin{array}{l}\text { Statuts } \\
(1854-1937) \\
\text { Registre du } \\
\text { commerce } \\
(1941-1946) \\
\text { Liquidation } \\
(1944-1953) \\
\\
\text { (4 articles) }\end{array}$ & $\begin{array}{l}\text { Statuts } \\
(1854-1952) \\
\text { Registre du } \\
\text { commerce } \\
(1919-1946) \\
\text { État des actionnaires } \\
(1945) \\
\\
(1 \text { article) }\end{array}$ \\
\hline 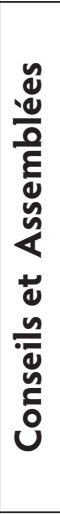 & 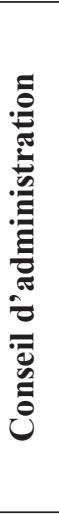 & $\begin{array}{l}\text { PV de réunions } \\
\text { (1856-1948) } \\
\text { Rapports, } \\
\text { comptes-rendus } \\
\text { d'exercice } \\
(1854-1944)\end{array}$ & \begin{tabular}{|l} 
PV de réunions \\
$(1846-1854)$ \\
Rapports des \\
administrateurs- \\
délégués \\
$(1849-1854)$
\end{tabular} & $\begin{array}{l}\text { PV de réunions } \\
\text { (1854-1946) } \\
\text { Rapports } \\
\text { (1854-1928) }\end{array}$ & $\begin{array}{l}\text { PV de réunions } \\
\text { (1927-1929 et } \\
\text { 1941-1946) }\end{array}$ & $\begin{array}{l}\text { PV de réunions } \\
\text { (1854-1946) } \\
\text { Rapports du } \\
\text { directeur } \\
\text { (1855-1944) } \\
\text { Lettres du direc- } \\
\text { teur au président } \\
\text { (1896-1931) } \\
\text { Secrétariat du } \\
\text { conseil } \\
\text { (1900-1945) } \\
\text { (14 articles) }\end{array}$ \\
\hline & 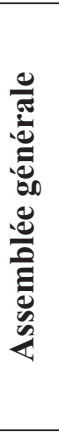 & $\begin{array}{l}\text { PV de réunions } \\
\text { (1850-1948) } \\
\text { Rapports du CA } \\
(1873-1886) \\
\text { Comptes présentés } \\
(1895-1945) \\
\text { Différend porté } \\
\text { au tribunal arbitral } \\
\text { (1923) } \\
\text { (9 articles) } \\
\end{array}$ & \begin{tabular}{|l|} 
PV de réunions \\
$(1844-1854)$ \\
Rapports du CA \\
$(1847-1854)$
\end{tabular} & $\begin{array}{l}\text { PV de réunions } \\
\text { (1941-1949) } \\
\text { Comptes rendus } \\
\text { imprimés } \\
\text { (1854-1946) } \\
\\
\text { (11 articles) }\end{array}$ & \begin{tabular}{|l}
$\begin{array}{l}\text { Rapports et } \\
\text { décisions } \\
(1854-1945)\end{array}$ \\
\\
(6 articles) \\
\end{tabular} & $\begin{array}{l}\text { PV de réunions } \\
\text { (1855-1945) } \\
\text { Rapports } \\
\text { (1855-1945) } \\
\text { Organisation des } \\
\text { assemblées } \\
\text { (1928-1948) } \\
\\
\text { (9 articles) } \\
\end{array}$ \\
\hline & 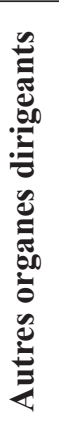 & & $\begin{array}{l}\text { Différents } \\
\text { comités et } \\
\text { commissions } \\
\text { PV de réunions } \\
(1844-1854) \\
\\
\\
\text { (8 articles) }\end{array}$ & & \begin{tabular}{|l|} 
Comité de direction \\
PV de réunions \\
(1925-1929) \\
\\
(1 article)
\end{tabular} & \\
\hline
\end{tabular}




\begin{tabular}{|c|c|c|c|c|c|}
\hline 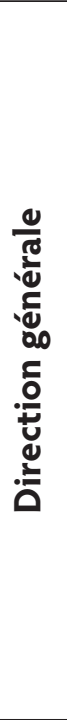 & 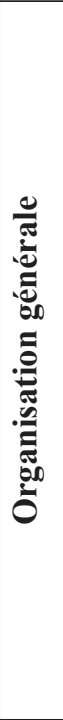 & $\begin{array}{l}\text { Pouvoirs délégués } \\
\text { au directeur } \\
\text { (1927-1946) } \\
\text { Règlements } \\
\text { généraux } \\
\text { d'exploitation } \\
\text { (1895-1919) } \\
\text { Automobile du } \\
\text { directeur } \\
\text { Notes de service } \\
\text { (1874-1946) }\end{array}$ & $\begin{array}{l}\text { Rapports annuels } \\
\text { des ingénieurs } \\
\text { divisionnaires } \\
(1911-1921) \\
\text { Notes de service } \\
\text { (1906-1945) } \\
\text { Statistiques mensuel- } \\
\text { les d'exploitation } \\
\text { (1911-1944) } \\
\text { Statistiques sociales } \\
\text { (1857-1913) } \\
\text { Correspondance } \\
\text { avec le Conseil } \\
\text { ou le président } \\
\text { (1855-1946) } \\
\text { Visites des puits } \\
\text { (123 articles) }\end{array}$ & $\begin{array}{l}\text { Réforme de la } \\
\text { législation minière } \\
\text { (1875-1944) } \\
\\
\text { (1 article) }\end{array}$ & $\begin{array}{l}\text { Pouvoirs } \\
\text { délégués au } \\
\text { directeur } \\
\text { (1914-1946) } \\
\text { Correspondance } \\
\text { reçue du Conseil } \\
\text { (1874-1946) } \\
\text { Notes de service } \\
\text { (1930-1946) } \\
\text { Rapports, } \\
\text { statistiques, } \\
\text { dossiers du } \\
\text { directeur } \\
\text { (1930-1945) } \\
\text { Visites des puits } \\
\text { (1929-1943) } \\
\text { (13 articles) }\end{array}$ \\
\hline & 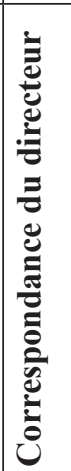 & & $\begin{array}{l}\text { (1854-1934) } \\
\text { (18 articles) }\end{array}$ & & (1900-1941) \\
\hline & 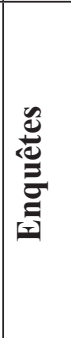 & 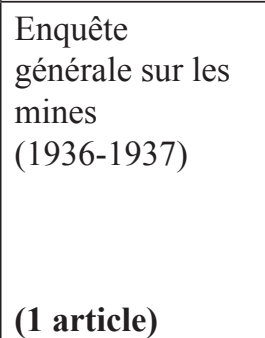 & $\begin{array}{l}\text { Enquête sur la } \\
\text { situation } \\
\text { financière } \\
\text { (1936-1937) } \\
\text { (1 article) }\end{array}$ & & $\begin{array}{l}\text { Études économiques } \\
\text { (1873-1933) } \\
\text { Commission } \\
\text { d'études du coût } \\
\text { de la vie } \\
\text { (1921-1939) } \\
\text { (2 articles) }\end{array}$ \\
\hline
\end{tabular}




\begin{tabular}{|c|c|c|c|c|}
\hline 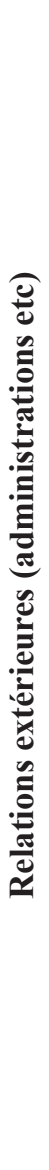 & $\begin{array}{l}\text { Relations avec } \\
\text { différentes } \\
\text { administrations } \\
\text { (1853-1917) } \\
\text { Relations avec } \\
\text { l'administration } \\
\text { des Mines } \\
\text { (1901-1946) } \\
\text { Relations avec } \\
\text { les municipalités } \\
\text { (1855-1907) } \\
\text { Relations avec } \\
\text { associations } \\
\text { (1929-1941) } \\
\text { Radio-reportage } \\
\text { (radio de Lyon) } \\
\text { (1931) } \\
\end{array}$ & $\begin{array}{l}\text { Statistiques desti- } \\
\text { nées à l'adminis- } \\
\text { tration des mines } \\
(1904-1914) \\
\text { Relations avec } \\
\text { l'administration } \\
\text { des mines } \\
\text { (1867-1946) }\end{array}$ & $\begin{array}{l}\text { Relations avec } \\
\text { l'administration } \\
\text { des mines } \\
(1913-1946)\end{array}$ & $\begin{array}{l}\text { Réglementation } \\
\text { de l'exploitation } \\
\text { (1872-1912) } \\
\text { Relations avec } \\
\text { l'administration } \\
\text { des Mines } \\
\text { (1903-1946) } \\
\text { Relations avec } \\
\text { d'autres } \\
\text { administrations } \\
\text { (1845-1956) } \\
\text { Relations avec } \\
\text { les municipalités } \\
\text { (1898-1938) } \\
\text { École des Mines } \\
\text { de Saint-Étienne } \\
\text { (1888-1946) } \\
\text { Conseil supérieur } \\
\text { des voies de com- } \\
\text { munication (1878) } \\
\text { Pollution des eaux } \\
\text { (1925-1938) } \\
\text { Associations } \\
\text { (SPA, anciens élè- } \\
\text { ves de l'École des } \\
\text { Mines) } \\
\text { (1886-1936) } \\
\text { (17 articles) }\end{array}$ \\
\hline 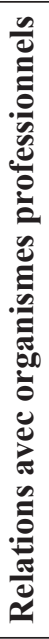 & $\begin{array}{l}\text { Comité des } \\
\text { houillères et } \\
\text { autres organismes } \\
\text { miniers } \\
(1927-1949) \\
\text { Exposition } \\
\text { (Lyon 1893) } \\
\\
\text { (5 articles) }\end{array}$ & 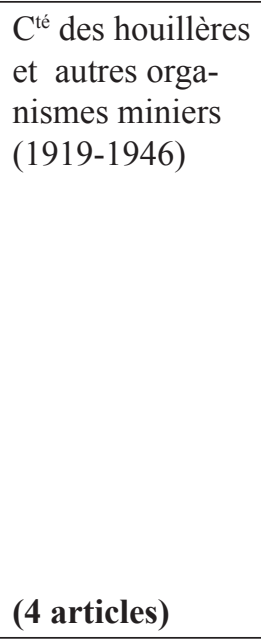 & $\begin{array}{l}\text { Syndicat patronal } \\
(1944) \\
\text { Compensation des } \\
\text { recettes minières } \\
(1944-1946) \\
\\
(2 \text { articles) }\end{array}$ & $\begin{array}{l}C^{\text {tés }} \text { des houillères } \\
\text { et autres orga- } \\
\text { nismes miniers } \\
\text { (1904-1943) } \\
\text { Chambres de } \\
\text { commerce } \\
\text { (1922-1933) } \\
\text { Expositions } \\
\text { universelles } \\
\text { (1865-1900) } \\
\text { Voyages d'étude } \\
\text { (1887-1939) } \\
\text { (13 articles) }\end{array}$ \\
\hline 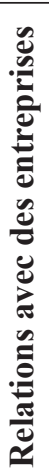 & & $\begin{array}{l}(1857-1946) \\
\text { (4 articles) }\end{array}$ & $(1853-1950)$ & (1 article) \\
\hline
\end{tabular}




\begin{tabular}{|c|c|c|c|c|c|c|}
\hline & 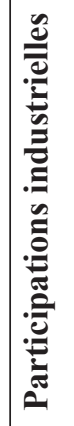 & (1 article) & & $\begin{array}{l}(1929-1945) \\
\text { (2 articles) }\end{array}$ & & $\begin{array}{l}\text { (1912-1945) } \\
\text { (9 articles) }\end{array}$ \\
\hline & 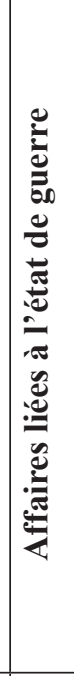 & $\begin{array}{l}\text { Mobilisation } \\
(1929-1940) \\
\text { Protection civile } \\
(1939-1940) \\
\text { Communications } \\
\text { téléphoniques } \\
(1939-1941) \\
\text { Réquisitions, } \\
\text { travaux pour } \\
\text { l'armée } \\
(1940-1941) \\
\text { Relations } \\
\text { économiques } \\
\text { avec l'occupant } \\
\text { (1942-1945) } \\
(\mathbf{9} \text { articles) }\end{array}$ & & $\begin{array}{l}\text { Défense passive } \\
\text { (1943-1946) } \\
\text { Prisonniers } \\
\text { allemands } \\
\text { (1944-1947) } \\
\\
\text { (4 articles) }\end{array}$ & $\begin{array}{l}\text { Révision des } \\
\text { marchés de guerre } \\
(1933-1944) \\
\text { Relations avec } \\
\text { l'occupant } \\
(1944-1949) \\
\text { Dommages de } \\
\text { guerre } \\
(1940-1944) \\
\\
\text { (3 articles) } \\
\end{array}$ & $\begin{array}{l}\text { Dommages } \\
\text { de guerre } \\
(1940-1948)\end{array}$ \\
\hline & 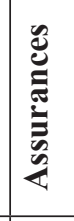 & $\begin{array}{l}\text { (1926-1948) } \\
\text { (3 articles) } \\
\end{array}$ & & & $\begin{array}{l}(1921-1945) \\
(1 \text { article) } \\
\end{array}$ & \\
\hline 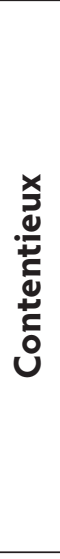 & & $\begin{array}{l}\text { (dégâts de sur- } \\
\text { face, accidents du } \\
\text { travail, conten- } \\
\text { tieux commercial) } \\
(1861-1940) \\
\\
\\
\text { (5 articles) } \\
\end{array}$ & $\begin{array}{l}\text { (dégâts de } \\
\text { surface, redevan- } \\
\text { ce proportion- } \\
\text { nelle, droits } \\
\text { d'exploitation) } \\
\text { (1841-1856) } \\
\\
\text { (7 articles) } \\
\end{array}$ & $\begin{array}{l}\text { Sommier, copies } \\
\text { de lettres } \\
(1848-1943) \\
\text { Droits d'exploi- } \\
\text { tation } \\
(1849-1919) \\
\text { Dégâts de surface } \\
(1851-1921) \\
\text { Accidents du } \\
\text { travail } \\
(1908-1909) \\
\text { (63 articles) } \\
\end{array}$ & & $\begin{array}{l}\text { (droits d'exploi- } \\
\text { tation, droits tré- } \\
\text { fonciers, dégâts de } \\
\text { surface) } \\
(1824-1925)\end{array}$ \\
\hline 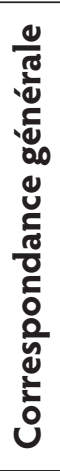 & & 更 & 更 & (101 articles) & \begin{tabular}{|l}
$(1903-1936)$ \\
\\
(9 articles)
\end{tabular} & (25 articles) \\
\hline
\end{tabular}




\begin{tabular}{|c|c|c|c|c|c|c|}
\hline 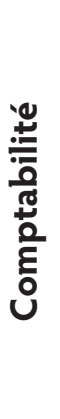 & 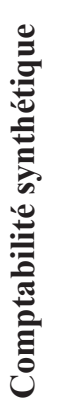 & $\begin{array}{l}\text { Bilans (lacunes) } \\
(1876-1946) \\
\text { Inventaires } \\
(1900-1943) \\
\text { Balances } \\
(1933-1948)\end{array}$ & & $\begin{array}{l}\text { Bilans (lacunes) } \\
\text { (1902-1949) } \\
\text { Inventaires } \\
\text { (1927-1945) } \\
\text { Balances } \\
\text { (1939-1946) } \\
\\
\text { (12 articles) }\end{array}$ & $\begin{array}{l}\text { Bilans (lacunes) } \\
(1864-1946) \\
\text { Inventaires } \\
(1936-1946) \\
\text { Balances } \\
(1936-1946)\end{array}$ & $\begin{array}{l}\text { Bilans (1932- } \\
\text { 1935, 1943-1948) } \\
\text { Statistiques } \\
\text { (lacunes) } \\
\text { (1854-1953) } \\
\text { Situations } \\
\text { financières } \\
\text { (1918-1946) } \\
\text { (25 articles) }\end{array}$ \\
\hline & 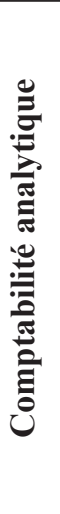 & $\begin{array}{l}\text { Grand livre } \\
(1850,1938- \\
1946) \\
\text { Journaux } \\
\text { (1820-1946)(lacunes) } \\
\text { Livres de paye } \\
\text { (1937-1944) } \\
\text { Registres des } \\
\text { recettes et dépen- } \\
\text { ses par secteurs } \\
\text { (1913-1946) } \\
\text { (152 articles) }\end{array}$ & $\begin{array}{l}\text { Journal général } \\
\text { (1844-1845) } \\
\text { Main d'œuvre } \\
\text { et fournitures } \\
\text { (1846-1855) } \\
\text { (49 articles) }\end{array}$ & \begin{tabular}{|l} 
Grand livre \\
(1930-1946) \\
Journal général \\
$(1854-1946)$ \\
(lacunes) \\
Journal des ventes \\
(1927-1946) \\
Livres de paye \\
(1888-1938) \\
(lacunes) \\
\\
(117+661 articles)
\end{tabular} & $\begin{array}{l}\text { Grand livre } \\
(1910-1950) \\
\text { Journal des ventes } \\
(1932-1946)\end{array}$ & $\begin{array}{l}\text { Grand livre } \\
(1854-1946) \\
\text { Journaux } \\
\text { (1854-1946) } \\
\text { (lacunes) } \\
\text { Livres de paye } \\
\text { (1853-1922) } \\
\text { (lacunes) } \\
\\
\text { (92 + 1086 articles) }\end{array}$ \\
\hline 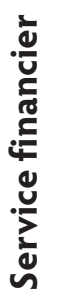 & 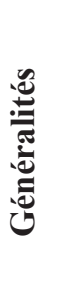 & $\begin{array}{l}\text { Enquête de } \\
\text { l'inspection des } \\
\text { finances } \\
(1931-1937) \\
\text { (1 article) }\end{array}$ & & $\begin{array}{l}\text { Correspondance } \\
(1920-1946)\end{array}$ & $\begin{array}{l}\text { Correspondance } \\
(1942-1949) \\
\text { Règlement de } \\
\text { créances (liquida- } \\
\text { tion judiciaire) } \\
\text { (1921-1950) } \\
\text { (15 articles) }\end{array}$ & $\begin{array}{l}\text { Fonctionnement } \\
\text { du service } \\
(1917-1920)\end{array}$ \\
\hline & 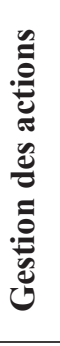 & (5 articles) & & & $\begin{array}{l}(1942-1943) \\
\text { (1 article) }\end{array}$ & \\
\hline & 氞 & $\begin{array}{l}\text { Journaux et } \\
\text { inventaires du } \\
\text { portefeuille } \\
(1899-1952) \\
(7 \text { articles) }\end{array}$ & & $\begin{array}{l}\begin{array}{l}\text { États annuels } \\
(1894-1948)\end{array} \\
\text { (3 articles) } \\
\end{array}$ & (1 article) & $(1904-1950)$ \\
\hline & 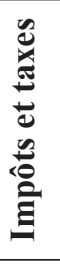 & $(1910-1950)$ & & $(1922-1947)$ & $(1920-1951)$ & $(1916-1950)$ \\
\hline
\end{tabular}




\begin{tabular}{|c|c|c|c|c|c|}
\hline & 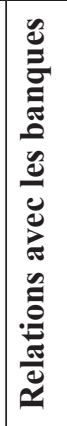 & $\begin{array}{l}(1931-1936) \\
\text { (2 articles) }\end{array}$ & & $\begin{array}{l}(1942-1946) \\
\text { (2 articles) }\end{array}$ & $\begin{array}{l}\text { (1923-1930) } \\
\text { (1 article) }\end{array}$ \\
\hline & 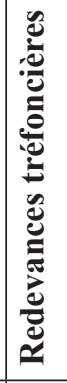 & & $\begin{array}{l}\text { Comptes courants } \\
(1895-1934) \\
\text { Calcul des } \\
\text { redevances } \\
(1884-1945) \\
\\
\text { (10 articles) }\end{array}$ & \begin{tabular}{|l}
$(1931-1948)$ \\
\\
(5 articles) \\
\end{tabular} & (1 article) \\
\hline 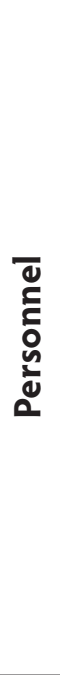 & 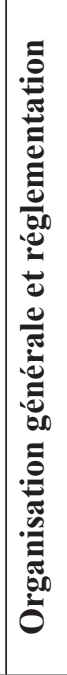 & \begin{tabular}{|l} 
Règlements \\
généraux \\
$(1905-1934)$ \\
Conventions \\
collectives \\
$(1936-1942)$ \\
Organisation \\
scientifique \\
$(1932-1937)$ \\
Contrôle et \\
surveillance \\
(1917-1949) \\
Travail des femmes \\
et des enfants \\
(1892-1932) \\
(8 articles)
\end{tabular} & $\begin{array}{l}\text { Correspondance } \\
\text { (1906-1911) } \\
\text { Dossiers individuels } \\
\text { (ingénieurs, } \\
\text { employés) } \\
\text { (1930-1945) } \\
\text { Reclassement de } \\
\text { mineurs licenciés } \\
\text { (1932) }\end{array}$ & $\begin{array}{l}\text { Copies de lettres } \\
(1929-1936) \\
\text { Inscription des } \\
\text { livrets } \\
(1857-1900) \\
\\
\\
\text { (10 articles) }\end{array}$ & $\begin{array}{l}\text { Règlements } \\
\text { généraux } \\
(1899-1945) \\
\text { Gestion générale } \\
(1898-1945) \\
\text { Organisation } \\
\text { scientifique } \\
(1929-1940) \\
\text { Semaine de } 40 \text { h. } \\
\text { (1936) } \\
\text { Fautes } \\
\text { professionnelles } \\
\text { (1931-1944) } \\
\\
\text { (7 articles) }\end{array}$ \\
\hline & 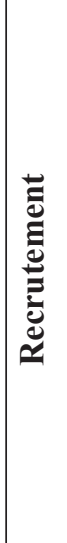 & $\begin{array}{l}\text { Employés } \\
\text { (1944-1947) } \\
\text { Main d'œuvre } \\
\text { féminine } \\
(1942-1946) \\
\text { Main d'œuvre } \\
\text { coloniale } \\
\text { (1936-1946) } \\
\text { Main d'œuvre } \\
\text { étrangère } \\
\text { (1926-1953) } \\
\text { (4 articles) }\end{array}$ & $\begin{array}{l}\text { Ingénieurs } \\
\text { (1906-1911) } \\
\text { Main d'œuvre } \\
\text { étrangère } \\
\text { (1931) } \\
\text { (1 article) }\end{array}$ & & $\begin{array}{l}\text { Dossiers généraux } \\
(1898-1945) \\
\text { Examen d'entrée } \\
\text { (1941-1942) } \\
\text { Mutilés de guerre } \\
\text { (1920-1931) } \\
\text { Prisonniers de } \\
\text { guerre allemands } \\
\text { (1919-1947) } \\
\text { Main d'œuvre } \\
\text { étrangère et coloniale } \\
\text { (1919-1945) } \\
\text { (7 articles) }\end{array}$ \\
\hline
\end{tabular}




\begin{tabular}{|c|c|c|c|c|}
\hline 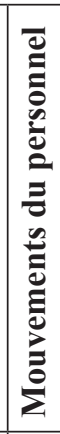 & \begin{tabular}{|l} 
Registres d'en- \\
trées et sorties \\
$(1906-1930)$ \\
\\
(7 articles)
\end{tabular} & & & $\begin{array}{l}(1910-1945) \\
\text { (5 articles) }\end{array}$ \\
\hline 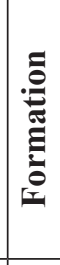 & & $\begin{array}{l}\text { Relations avec les } \\
\text { écoles } \\
\text { (1920-1935) } \\
\text { Apprentissage } \\
\text { (1941-1946) } \\
\text { (9 articles) } \\
\end{array}$ & & $\begin{array}{l}\text { Subventions } \\
(1943-1946)\end{array}$ \\
\hline 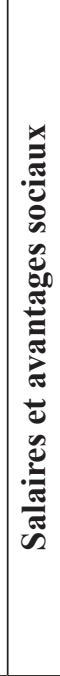 & \begin{tabular}{|l} 
Grilles \\
$(1919-1943)$ \\
Contrôles de \\
présence (par \\
puits) \\
$(1847-1918)$ \\
Fourniture de \\
charbon \\
$(1901-1946)$
\end{tabular} & $\begin{array}{l}\text { Contrôle de } \\
\text { présence (par } \\
\text { puits ou atelier) } \\
\text { (1889-1931) }\end{array}$ & $\begin{array}{l}\text { Allocations } \\
\text { annuelles } \\
(1930-1941) \\
\text { Appointements et } \\
\text { gratifications } \\
\text { (encadrement) } \\
\text { (1933-1934) } \\
\text { Charges sociales } \\
(1941-1946) \\
\text { Amendes } \\
\text { (1941-1952) } \\
\text { Comptes de } \\
\text { saisies-arrêts } \\
\text { (1939-1950) } \\
\text { (7 articles) }\end{array}$ & $\begin{array}{l}\text { Salaires ouvriers } \\
(1906-1945) \\
\text { Contrôle de } \\
\text { présence (par puits } \\
\text { ou atelier) } \\
\text { (lacunes) } \\
\text { (1854-1913) } \\
\text { Appointements } \\
\text { des ingénieurs } \\
\text { (1863-1940) } \\
\text { Allocations } \\
\text { chauffage } \\
(1914-1938) \\
\text { Secours } \\
(1900-1945) \\
\mathbf{( 8 + 1 0 8 1 ~ a r t i c l e s ) ~}\end{array}$ \\
\hline 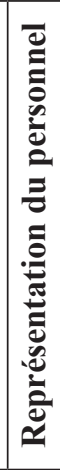 & \begin{tabular}{|l|} 
Syndicats \\
$(1936-1944)$ \\
Commission de \\
conciliation \\
$(1936-1939)$ \\
\\
\\
(2 articles)
\end{tabular} & & & (1 article) \\
\hline 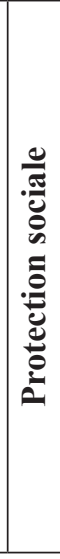 & $\begin{array}{l}\text { Caisses de secours, } \\
\text { allocations } \\
\text { familiales } \\
(1924-1946)\end{array}$ & $\begin{array}{l}\text { Caisses de secours, } \\
\text { allocations } \\
\text { familiales } \\
(1866-1948) \\
\\
\end{array}$ & & $\begin{array}{l}\text { Caisse de secours, } \\
\text { allocations } \\
\text { familiales } \\
\text { (1869-1946) } \\
\text { Assurance vie des } \\
\text { ingénieurs } \\
\text { (1882-1944) } \\
\text { Caisse de chô- } \\
\text { mage partiel } \\
\text { (1935) } \\
\text { Retraites } \\
\text { (1867-1947) } \\
\text { (16 articles) } \\
\end{array}$ \\
\hline
\end{tabular}




\begin{tabular}{|c|c|c|c|}
\hline 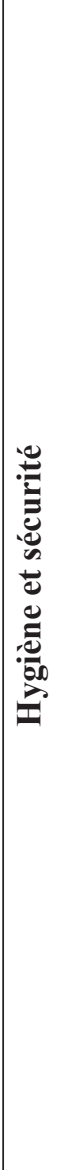 & $\begin{array}{l}\text { Consignes de } \\
\text { sécurité } \\
(1931-1942) \\
\text { Élection des } \\
\text { délégués mineurs } \\
(1870-1946) \\
\text { Visites des } \\
\text { délégués mineurs } \\
(1939-1942) \\
\text { Accidents du } \\
\text { travail } \\
(1878-1946) \\
\text { Catastrophe du } \\
\text { puits Combes } \\
\text { (1928-1933) }\end{array}$ & $\begin{array}{l}\text { Surveillance } \\
\text { médicale des } \\
\text { jeunes ouvriers } \\
(1941-1942) \\
\text { Maladies } \\
\text { professionnelles } \\
\text { (1916-1945) } \\
\text { Prévention des } \\
\text { accidents } \\
\text { (1930-1937) } \\
\text { Délégués mineurs } \\
\text { (rapports de } \\
\text { visites) } \\
\text { (1890-1915, } \\
\text { 1944-1948) } \\
\text { Visites de grisou } \\
\text { (1895-1910) } \\
\text { Assurances contre } \\
\text { les accidents } \\
\text { (1934-1946) } \\
\text { Accidents du } \\
\text { travail } \\
\text { (1893-1915) } \\
\text { Catastrophes } \\
\text { (Puits de la Loire, } \\
\text { La Chana) } \\
\text { (1939-1945) } \\
\text { (18 articles) }\end{array}$ & $\begin{array}{l}\text { Règlements } \\
\text { généraux } \\
(1824-1945) \\
\text { Prévention des } \\
\text { accidents et } \\
\text { sauvetage } \\
\text { (1906-1945) } \\
\text { Délégués mineurs } \\
\text { (élections, } \\
\text { rapports) } \\
\text { (1890-1946) } \\
\text { Procès-verbaux ou } \\
\text { relevés } \\
\text { d'accidents } \\
\text { (1890-1946) } \\
\text { Indemnités aux } \\
\text { blessés } \\
\text { (1905-1915) }\end{array}$ \\
\hline 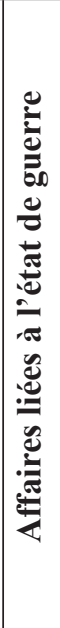 & $\begin{array}{l}\text { Mobilisation du } \\
\text { personnel et de } \\
\text { la main d'œuvre } \\
\text { étrangère } \\
\text { (1939-1944) } \\
\text { Affectation } \\
\text { spéciale des } \\
\text { ingénieurs } \\
(1939-1940) \\
\text { Réfugiés } \\
\text { (1939-1940) } \\
\text { Mineurs prisonniers } \\
\text { (1939-1940) } \\
\text { (5 articles) }\end{array}$ & $\begin{array}{l}\text { Monument aux } \\
\text { morts 1914-1918 } \\
(1920) \\
\\
\text { (1 article) }\end{array}$ & $\begin{array}{l}\text { Morts au champ } \\
\text { d'honneur, décorés } \\
\text { de la Croix de } \\
\text { Guerre } \\
\text { (1918-1928) } \\
\text { Mobilisation des } \\
\text { employés } \\
\text { (1921-1946) } \\
\text { Réquisition, STO, } \\
\text { déportation } \\
\text { (1939-1945) } \\
\text { Indemnisation des } \\
\text { heures perdues } \\
\text { (1944-1945) } \\
\text { (5 articles) }\end{array}$ \\
\hline
\end{tabular}




\begin{tabular}{|c|c|c|c|c|c|c|}
\hline 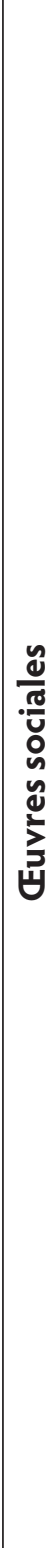 & & $\begin{array}{l}\text { Comité social } \\
(1941-1945) \\
\text { Service social } \\
(1941-1949) \\
\text { Dispensaires, } \\
\text { religieuses } \\
\text { hospitalières } \\
(1927-1945) \\
\text { Bureau de consul- } \\
\text { tation juridique } \\
(1927-1930) \\
\text { Colonies de } \\
\text { vacances et } \\
\text { maison de jeunes } \\
(1928-1944) \\
\text { Écoles } \\
(1882-1946) \\
\text { Logements } \\
\text { ouvriers } \\
(1894-1946) \\
\text { Jardins ouvriers } \\
(1927-1946) \\
\text { Coopérative } \\
(1932-1945) \\
\text { Organismes de } \\
\text { loisirs } \\
(1931-1944) \\
\text { Relations avec } \\
\text { des organismes } \\
\text { extérieurs } \\
(1892-1947) \\
\end{array}$ & & $\begin{array}{l}\text { Prix de vertu } \\
(1928-1946) \\
\text { Amicale des } \\
\text { retraités } \\
(1932-1945) \\
\text { Associations et } \\
\text { équipements } \\
\text { sportifs } \\
(1931-1943) \\
\text { Colonies de } \\
\text { vacances } \\
\text { (1941-1948) } \\
\text { Logements } \\
\text { ouvriers } \\
(1913-1928) \\
\end{array}$ & $\begin{array}{l}\text { Hospice } \\
(1870-1903) \\
\text { Colonies de } \\
\text { vacances } \\
(1941-1946) \\
\text { Solidarité aux } \\
\text { victimes du bom- } \\
\text { bardement de } 1944 \\
(1944-1945)\end{array}$ & $\begin{array}{l}\text { Comité social } \\
(1941-1944) \\
\text { Service social } \\
(1941-1947) \\
\text { Hospices et } \\
\text { dispensaires du } \\
\text { Montcel } \\
(1884-1945) \\
\text { Colonies de } \\
\text { vacances } \\
(1941-1943) \\
\text { Scouts } \\
\text { (1942-1945) } \\
\text { École du Montcel } \\
(1895-1947) \\
\text { Écoles de cadres } \\
(1943-1944) \\
\text { Associations } \\
\text { sportives } \\
\text { (1920-1944) } \\
\text { Associations } \\
\text { artistiques } \\
(1942-1944) \\
\text { Logements } \\
\text { ouvriers } \\
(1904-1946) \\
\text { Jardins ouvriers } \\
(1940-1946) \\
\text { CEuvres polonaises } \\
(1922-1945) \\
\text { Sociétés de secours } \\
\text { mutuels } \\
(1895-1906) \\
\text { Subventions à des } \\
\text { associations } \\
(1900-1945) \\
\text { (27 articles) }\end{array}$ \\
\hline 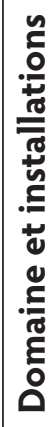 & 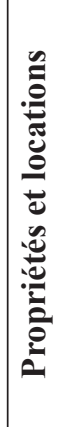 & $\begin{array}{l}\text { Acquisition et } \\
\text { cession de } \\
\text { terrains } \\
(1772-1946) \\
\text { Domaine forestier } \\
(1924-1927) \\
\\
\text { (8 articles) }\end{array}$ & $\begin{array}{l}\text { Achats ou } \\
\text { locations de } \\
\text { terrains ou } \\
\text { immeubles } \\
\text { (1831-1856) } \\
\\
\text { (1 article) }\end{array}$ & $\begin{array}{l}\text { Propriétés ; } \\
\text { locations, } \\
\text { expropriations } \\
(1882-1949)\end{array}$ & 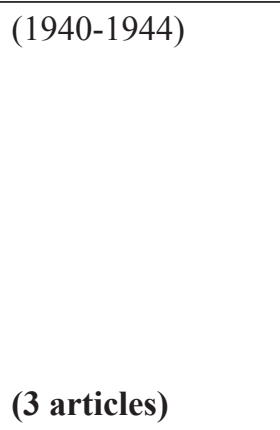 & \begin{tabular}{|l} 
Inventaire \\
$(1945)$ \\
Achats et ventes \\
$(1872-1946)$ \\
Domaines \\
forestiers \\
(propriété et \\
exploitation) \\
$(1890-1950)$ \\
$(\mathbf{3}+\mathbf{3 8}$ articles)
\end{tabular} \\
\hline & 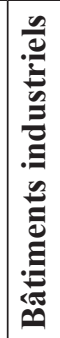 & & & $\begin{array}{l}\text { Photographies des } \\
\text { installations de } \\
\text { surface } \\
(1913-1941)\end{array}$ & & \\
\hline
\end{tabular}




\begin{tabular}{|c|c|c|c|c|c|c|}
\hline & 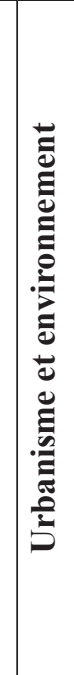 & \begin{tabular}{|l} 
Subventions \\
industrielles aux \\
chemins \\
$(1846-1942)$ \\
Relations avec \\
la commune de \\
Firminy \\
$(1904-1946)$ \\
Enquête avant- \\
projet de canal de \\
navigation de la \\
Loire au Rhône \\
(1882-1919) \\
Pollution des \\
eaux (1927-1946) \\
(4 articles)
\end{tabular} & & & & $\begin{array}{l}\text { Relations avec les } \\
\text { services vicinaux } \\
\text { (1851-1946) } \\
\\
\text { (1 article) }\end{array}$ \\
\hline & 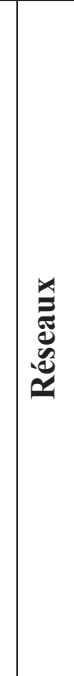 & $\begin{array}{l}\text { Eau } \\
(1877-1948) \\
\text { Électricité } \\
(1925-1929) \\
\\
\\
(3 \text { articles) }\end{array}$ & $\begin{array}{l}\text { Réglementation } \\
\text { spécifique } \\
(1861-1945) \\
\text { Relations avec } \\
\text { les Cies } \\
\text { (contentieux) } \\
(1859-1955) \\
\text { Installation des } \\
\text { lignes } \\
\text { (1840-1951) } \\
\text { Exploitation } \\
\text { (taxes et tarifs, } \\
\text { journaux de } \\
\text { transport) } \\
\text { (1923-1946) } \\
\text { (14 articles) }\end{array}$ & \begin{tabular}{|l} 
Relations avec \\
la $C^{\text {ie }}$ \\
$(1828-1859)$ \\
Installation d'une \\
ligne \\
$(1851-1853)$ \\
\\
\\
(3 articles)
\end{tabular} & & $\begin{array}{l}\text { Eau } \\
(1850-1944) \\
\text { Électricité } \\
\text { (1915-1944) } \\
\text { Téléphone } \\
\text { (1923-1934) } \\
\\
\\
\text { (4 articles) }\end{array}$ \\
\hline & 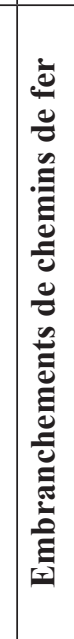 & \begin{tabular}{|l} 
Réglementation \\
spécifique \\
$(1861-1945)$ \\
Relations avec les \\
Cies (contentieux) \\
$(1859-1955)$ \\
Installation des \\
lignes \\
$(1840-1951)$ \\
Exploitation \\
(taxes et tarifs, \\
journaux de \\
transport) \\
$(1923-1946)$ \\
(14 articles)
\end{tabular} & $\begin{array}{l}\text { Relations avec } \\
\text { la } C^{\text {ie }} \\
(1828-1859) \\
\text { Installation d'une } \\
\text { ligne } \\
(1851-1853) \\
\\
\text { (3 articles) }\end{array}$ & & & $\begin{array}{l}\text { Relations avec les } \\
\text { Cies (contentieux) } \\
(1861-1868) \\
\text { Installation des } \\
\text { lignes } \\
(1860-1929) \\
\\
\text { (2 articles) }\end{array}$ \\
\hline 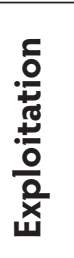 & 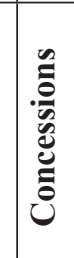 & (6 articles) & & $\begin{array}{l}(1864-1931) \\
\text { (5 articles) }\end{array}$ & $\begin{array}{l}\text { Amodiations } \\
(1925-1946) \\
\\
\text { (4 articles) }\end{array}$ & \\
\hline
\end{tabular}




\begin{tabular}{|c|c|c|c|c|c|}
\hline 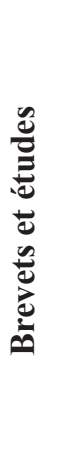 & $\begin{array}{l}\text { Brevets } \\
(1929-1947) \\
\text { Rapports et } \\
\text { études techniques } \\
(1887-1935) \\
\text { Voyages } \\
\text { d'ingénieurs } \\
(1902-1925) \\
\text { Congrès } \\
(1922-1942) \\
\text { (8 articles) }\end{array}$ & $\begin{array}{l}\text { Rapports } \\
\text { techniques } \\
(1846-1851) \\
\\
\text { (3 articles) }\end{array}$ & & & $\begin{array}{l}\text { Service des } \\
\text { études et du } \\
\text { matériel (rapports } \\
\text { mensuels) } \\
(1905-1946) \\
\\
\text { (1 article) }\end{array}$ \\
\hline 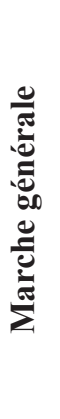 & $\begin{array}{l}\text { Cahiers annuels } \\
\text { des exploitations } \\
(1928-1946) \\
\text { Cahiers de statis- } \\
\text { tiques de } \\
\text { production } \\
(1920-1930) \\
\text { (10 articles) }\end{array}$ & & $\begin{array}{l}\text { Rapports } \\
\text { périodiques } \\
(1912-1943) \\
\text { Études des } \\
\text { réserves } \\
(1939-1944) \\
\text { Tableaux } \\
\text { comparatifs } \\
\text { (1856-1936) } \\
\text { (5 articles) }\end{array}$ & & $\begin{array}{l}\text { Rapports annuels } \\
\text { (1862-1945) } \\
\text { Statistiques } \\
\text { annuelles } \\
\text { (1933-1947) } \\
\\
\text { (17 articles) }\end{array}$ \\
\hline 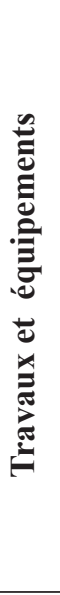 & $\begin{array}{l}(1867-1947) \\
\\
\text { (8 articles) }\end{array}$ & & $\begin{array}{l}\text { Programmation et } \\
\text { états des travaux } \\
\text { (1904-1947) } \\
\text { (10 articles) }\end{array}$ & $\begin{array}{l}\text { Copies de lettres } \\
\text { (1929-1942) } \\
\text { Relation avec une } \\
\text { entreprise } \\
(1937-1945)\end{array}$ & $\begin{array}{l}\text { Résumés et états } \\
\text { de travaux } \\
(1886-1933) \\
\text { Tableaux } \\
\text { statistiques } \\
(1908-1935) \\
\text { Comptes rendus } \\
\text { mensuels } \\
\text { d'avancement } \\
\text { (1914-1923) } \\
\text { Électrification } \\
\text { (1896-1923) } \\
\text { Aérage (1866) } \\
\text { (10 articles) }\end{array}$ \\
\hline 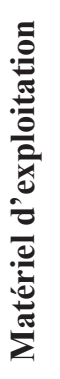 & (4 articles) & & & & $\begin{array}{l}\text { Machines } \\
(1918-1946)\end{array}$ \\
\hline
\end{tabular}




\begin{tabular}{|c|c|c|c|c|c|}
\hline & 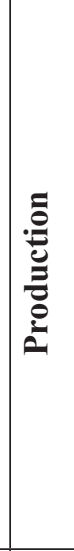 & \begin{tabular}{|l} 
Cahiers de \\
production \\
$(1920-1930$ \\
Journaux \\
industriels \\
$(1928-1945)$ \\
Registres des \\
stocks \\
$(1933-1945)$ \\
Registres des prix \\
de revient \\
$(1931-1949)$ \\
$\mathbf{( 4 9}$ articles) \\
\end{tabular} & $\begin{array}{l}\text { Prix de revient } \\
\text { (1939-1948) } \\
\text { Comptes de } \\
\text { piquage } \\
\text { (1908-1948) }\end{array}$ & $\begin{array}{l}\text { Tableaux annuels } \\
\text { (1942-1943) } \\
\text { Prix de revient } \\
(1929-1936) \\
\\
\text { (3 articles) } \\
\end{array}$ & $\begin{array}{l}\text { Prix de revient } \\
(1923-1946) \\
\text { État des stocks } \\
(1944-1953) \\
\text { Commission du } \\
\text { nouveau puits } \\
\text { (1933-1940) } \\
\text { Journaux de mine } \\
\text { (1926-1940) } \\
\text { (15 articles) }\end{array}$ \\
\hline & 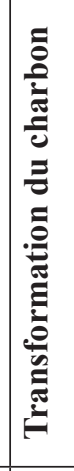 & & & & $\begin{array}{l}\text { Fours à coke } \\
(1910-1944)\end{array}$ \\
\hline 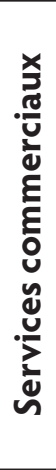 & & \begin{tabular}{|l} 
Fourniture au \\
ministère de la \\
Marine \\
$(1854-1891)$ \\
Fourniture de gaz \\
et d'électricité \\
$(1917-1943)$ \\
Registre des \\
acheteurs \\
$(1935-1945)$ \\
(7 articles)
\end{tabular} & $\begin{array}{l}\text { Correspondance } \\
\text { commerciale } \\
(1932-1938) \\
\text { États des ventes et } \\
\text { livres } \\
(1901-1944) \\
\\
\text { (13 articles) }\end{array}$ & $\begin{array}{l}\text { Ventes en } \\
\text { Belgique et Italie } \\
(1939-1945) \\
\\
\text { (2 articles) }\end{array}$ & $\begin{array}{l}\text { Accords } \\
\text { interentreprises } \\
\text { et organismes de } \\
\text { commercialisation } \\
(1914-1945) \\
\text { Fourniture de gaz } \\
\text { et d'électricité } \\
\text { (1922-1945) } \\
\text { (7 articles) }\end{array}$ \\
\hline
\end{tabular}

